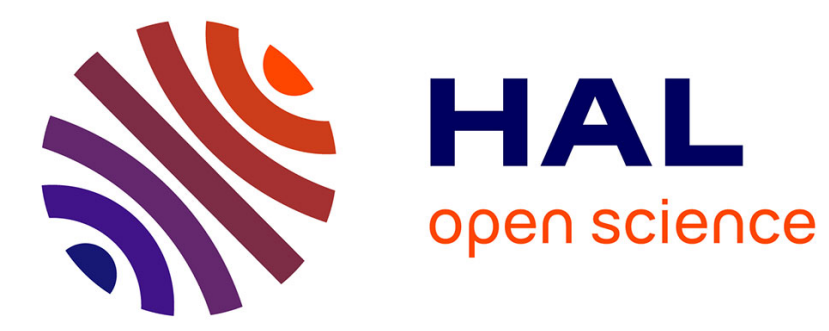

\title{
INTERACTION OF A SUBSURFACE BUBBLE LAYER WITH LONG INTERNAL WAVES
}

\author{
Sergey L. Gavrilyuk, Valery Liapidevskii, Alexander Chesnokov
}

\section{To cite this version:}

Sergey L. Gavrilyuk, Valery Liapidevskii, Alexander Chesnokov. INTERACTION OF A SUBSURFACE BUBBLE LAYER WITH LONG INTERNAL WAVES. European Journal of Mechanics B/Fluids, In press. hal-01712853

\section{HAL Id: hal-01712853 https://hal.science/hal-01712853}

Submitted on 19 Feb 2018

HAL is a multi-disciplinary open access archive for the deposit and dissemination of scientific research documents, whether they are published or not. The documents may come from teaching and research institutions in France or abroad, or from public or private research centers.
L'archive ouverte pluridisciplinaire HAL, est destinée au dépôt et à la diffusion de documents scientifiques de niveau recherche, publiés ou non, émanant des établissements d'enseignement et de recherche français ou étrangers, des laboratoires publics ou privés. 


\title{
INTERACTION OF A SUBSURFACE BUBBLE LAYER WITH LONG INTERNAL WAVES
}

\author{
Sergey Gavrilyuk ${ }^{\mathrm{a}, \mathrm{b}}$, Valery Liapidevskiib ${ }^{\mathrm{b}, \mathrm{c}}$, Alexander Chesnokov ${ }^{\mathrm{b}, \mathrm{c}}$ \\ ${ }^{a}$ Aix-Marseille Université, UMR CNRS 7343, IUSTI, 5 rue E. Fermi, 13453 Marseille CEDEX 13, France \\ ${ }^{b}$ Novosibirsk State University, 2 Pirogova Str., Novosibirsk 630090, Russia \\ ${ }^{c}$ Lavrentyev Institute of Hydrodynamics SB RAS, 15 Lavrentyev Ave., Novosibirsk 630090, Russia
}

\begin{abstract}
A two-layer model describing the interaction of a shear bubble layer formed by breaking waves and an underlying potential layer is derived in shallow water approximation. A nonhydrostatic formulation taking into account the entrainment effects in shear flows is proposed. Time and space periodic solutions are found, and some basic problems (the formation of bores and periodic structures from a uniform flow) are numerically solved.
\end{abstract}

Keywords: breaking waves; shear flows; two-layer shallow water flows.

\section{Introduction}

The wave breaking is a complex phenomenon accompanied by a strong vorticity generation, air entrainment and the interaction of a lower underlying layer (usually potential) under breaking waves and an upper subsurface shear layer [32]. The article by Duncan [7] provides a great overview of this phenomenon for spilling breakers. In $[2,3]$ a three-layer scheme for mathematical modelling of spilling breakers is proposed. It involves the underlying potential layer, intermediate single-phase turbulent layer, and subsurface two-phase layer. The dynamics of the frontiers between these physically different flow regions (potential, shear and multiphase) is one of the key points of the modelling [4, 20]. The model needs also to know the structure of the velocity profile in the shear region. A cubic approximation is used for this aim [19], [29]. The model was successfully used for the description of weak hydraulic jumps [21]. The influence of the internal waves on acoustic properties of subsurface bubble layer in a simplified framework was studied in [11].

This paper attempts to propose a mathematical model for the interaction between the bubble layer formed by breaking waves and internal waves (see Fig. 1). The flow in the upper bubble layer is considered as a shear flow of air-water mixture where the air bubbles and surrounding fluid have the same average velocity. Such a two-phase approach is largely used in a Navier-Stokes type modelling of breaking waves $[18,6]$. We neglect here both the

Email addresses: Corresponding author : sergey.gavrilyuk@univ-amu.fr (Sergey Gavrilyuk), liapid@hydro.nsc.ru (Valery Liapidevskii), chesnokov@hydro.nsc.ru (Alexander Chesnokov) 


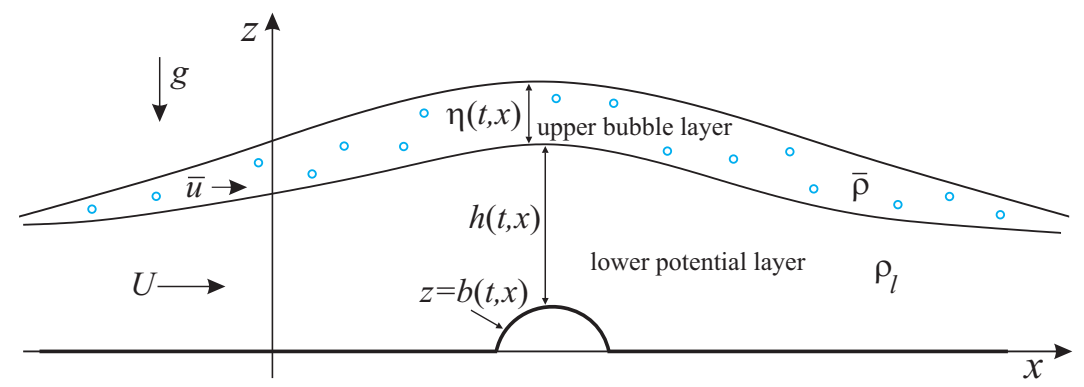

Figure 1: Two-layer stratified flow over topography.

viscosity and capillarity effects. The volume fraction will be the only parameter describing the presence of air bubbles in water. The flow is sheared, however, we do not suppose any specific dependence of the horiontal velocity on the vertical coordinate. Instead, under some mathematically justified hypotheses (shallow water approximation, smallness of the air volume fraction and its vertical gradient), we derive an evolution equation for the variable measuring the horizontal velocity profile distortion. This variable is naturally related to the flow vorticity. Then the depth averaging equations are derived. The upper-layer subsystem is hyperbolic, and this eventually leads to the formation of shocks in the solutions of the system. The vorticity is generated both by the shocks and entrainment process in shear flows (cf. [31, 24, 25, 5, 10]).

The lower homogeneous fluid layer is potential, and can approximately be described by the Serre-Su-Gardner-Green-Naghdi type equations [27, 28, 12]. The interaction between the layers is taken into account through a natural kinematic boundary condition at the interface "shear layer-potential layer" describing an entrapment of a pure fluid by a shear bubble flow. The entrainment velocity is proportional to the intensity of large eddies in the upper layer [33]. The presence of air drastically changes the flow characteristics. Indeed, the fluid with air bubbles is lighter than the below fluid, so the system becomes "stably stratified". The stratification induces buoyancy effects that are absent in homogeneous fluids. The model is a natural extension of the model [10] validated, in particular, on the propagation of Favre waves [8] and shoaling of solitary waves [14].

The paper is organized as follows. In sections 2 and 3 we derive two-layer depthaveraged equations for flows over time and space dependent topography. In section 4, a mild slope approximation of the model is proposed. Exact periodic solutions are constructed in section 5. In section 6 , a numerical method is presented together with the non-stationary computations showing the formation of a turbulent bore and periodic and damped solutions from a uniform flow. Technical details are presented in the Appendices A and B.

\section{Flow over topography for a lower layer with a prescribed pressure at the interface}

Consider the Euler equations for two-dimensional flows. In the horizontal direction $O x$ the component of the velocity is $u$, and in the vertical direction $O z$ the velocity component 
is $w$. With $\rho_{l}$ being the fluid density and $p$ being the fluid pressure, the Euler equations for the lower layer can be written as :

$$
\begin{gathered}
\frac{\partial u}{\partial x}+\frac{\partial w}{\partial z}=0 \\
\rho_{l}\left(\frac{\partial u}{\partial t}+u \frac{\partial u}{\partial x}+w \frac{\partial u}{\partial z}\right)=-\frac{\partial p}{\partial x} \\
\rho_{l}\left(\frac{\partial w}{\partial t}+u \frac{\partial w}{\partial x}+w \frac{\partial w}{\partial z}\right)=-\rho_{l} g-\frac{\partial p}{\partial z} .
\end{gathered}
$$

Here $g$ is the gravitational acceleration in the vertical direction. The kinematic boundary condition at $z=b(t, x)$ is :

$$
\left.w\right|_{z=b}=b_{t}+\left.u\right|_{z=b} b_{x} .
$$

At the internal boundary $z=b+h$ ( $h$ is the layer thickness) the kinematic condition is :

$$
\left.w\right|_{z=b+h}-(b+h)_{t}-\left.u\right|_{z=b+h}(b+h)_{x}=M .
$$

Here the right hand side $M$ is responsible for the mixing between layers (an empirical formula for $M$ will be proposed later).

We introduce the classical scaling of the shallow water theory:

$$
\begin{aligned}
& x=L x^{*}, \quad z=H z^{*}, \quad t=\frac{L}{\sqrt{g H}} t^{*}, \quad b=H b^{*}, \\
& u=\sqrt{g H} u^{*}, \quad w=\frac{H}{L} \sqrt{g H} w^{*}, \quad p=\rho_{l} g H p^{*}, \quad h=H h^{*} .
\end{aligned}
$$

Here $H$ and $L$ are the characteristic vertical and horizontal scales, the dimensionless variables are denoted with "star". We suppose that the waves are long, so the dimensionless parameter $\varepsilon=H / L$ is small. Equations (1)-(3) are transformed into dimensionless form where, to simplify the writing of equations, we drop the stars on the dimensionless variables:

$$
\begin{aligned}
\frac{\partial u}{\partial x}+\frac{\partial w}{\partial z} & =0 \\
\frac{\partial u}{\partial t}+\frac{\partial u^{2}}{\partial x}+\frac{\partial u w}{\partial z} & =-\frac{\partial p}{\partial x} \\
\varepsilon^{2}\left(\frac{\partial w}{\partial t}+\frac{\partial u w}{\partial x}+\frac{\partial w^{2}}{\partial z}\right) & =-\left(1+\frac{\partial p}{\partial z}\right) .
\end{aligned}
$$

Equations (6)-(8) admit the conservation of energy :

$$
\frac{\partial E}{\partial t}+\frac{\partial}{\partial x}(u E+p u)+\frac{\partial}{\partial z}(w E+p w)=0
$$

where

$$
E=\frac{u^{2}}{2}+\varepsilon^{2} \frac{w^{2}}{2}+z
$$


is the dimensionless specific energy. The dimensionless form of the boundary conditions (4) and (5) does not change under the hypothesis that the entrainment velocity $M$ has the same scaling as $w$.

Let us introduce the average velocity in the lower layer :

$$
U=\frac{1}{h} \int_{b}^{b+h} u d z
$$

Integrating equations (6), (7) and (9) with respect to $z$ over the fluid depth and using the boundary conditions, we get the following exact integral relations :

$$
\begin{gathered}
\frac{\partial h}{\partial t}+\frac{\partial}{\partial x}(h U)=-M \\
\frac{\partial}{\partial t}(h U)+\frac{\partial}{\partial x}\left(\int_{b}^{b+h} u^{2} d z+\int_{b}^{b+h} p d z\right) \\
=\left.p\right|_{z=b+h}(b+h)_{x}-\left.p\right|_{z=b} b_{x}-\left.M u\right|_{z=b+h}, \\
\left(\int_{b}^{b+h} E d z\right)_{t}+\left(\int_{b}^{b+h}(u E+p u) d z\right)_{x}=\left.p u\right|_{z=b+h}(b+h)_{x} \\
-\left.p u\right|_{z=b} b_{x}-\left.p w\right|_{z=b+h}+\left.p w\right|_{z=b}-\left.M E\right|_{z=b+h} .
\end{gathered}
$$

To obtain a closed system of equation, we need to know the pressure distribution in the layer. Integrating the equation (1) from $b$ to any $z, b<z<b+h$ we obtain :

$$
w(t, x, z)=\left.w\right|_{z=b}-\int_{b}^{z} u_{x} d z .
$$

In zero-order approximation the vertical velocity is :

$$
w(t, x, z) \approx \frac{D b}{D t}-U_{x}(z-b), \quad \frac{D}{D t}=\frac{\partial}{\partial t}+U \frac{\partial}{\partial x} .
$$

The second-order approximation for pressure comes from (8) where we have to replace $w$ by (13):

$$
\begin{aligned}
& \frac{\partial p}{\partial z}=-1-\varepsilon^{2}\left(\frac{\partial w}{\partial t}+u \frac{\partial w}{\partial x}+w \frac{\partial w}{\partial z}\right) \approx-1-\varepsilon^{2}\left(\frac{D w}{D t}+w \frac{\partial w}{\partial z}\right) \\
& =-1-\varepsilon^{2}\left(\frac{D^{2} b}{D t^{2}}-(z-b) \frac{D U_{x}}{D t}+U_{x} \frac{D b}{D t}-U_{x}\left(\frac{D b}{D t}-U_{x}(z-b)\right)\right) \\
& =-1-\varepsilon^{2}\left(\frac{D^{2} b}{D t^{2}}-(z-b)\left(\frac{D U_{x}}{D t}-U_{x}^{2}\right)\right) .
\end{aligned}
$$

We do not replace here $U_{x}$ from the mass conservation law (10) because of the source term $M$. Integrating (14) from $b+h$ to $z(b<z<b+h)$, we obtain:

$$
p=\left.p\right|_{z=b+h}-(z-b-h)-\varepsilon^{2} \frac{D^{2} b}{D t^{2}}(z-b-h)+\varepsilon^{2}\left(\frac{D U_{x}}{D t}-U_{x}^{2}\right) \frac{(z-b)^{2}-h^{2}}{2} .
$$


In particular, this gives the pressure distribution at the bottom:

$$
\left.p\right|_{z=b}=\left.p\right|_{z=b+h}+h+\varepsilon^{2} h \frac{D^{2} b}{D t^{2}}-\frac{\varepsilon^{2}}{2} h^{2}\left(\frac{D U_{x}}{D t}-U_{x}^{2}\right) .
$$

The integral of the pressure can then be evaluated as :

$$
P \equiv \int_{b}^{b+h} p d z=\left.p\right|_{z=b+h} h+\frac{h^{2}}{2}+\varepsilon^{2}\left(\frac{h^{2}}{2} \frac{D^{2} b}{D t^{2}}-\frac{h^{3}}{3}\left(\frac{D U_{x}}{D t}-U_{x}^{2}\right)\right) .
$$

We also introduce

$$
e=\frac{\varepsilon^{2}}{2 h} \int_{b}^{b+h} w^{2} d z
$$

Using (13) this "internal energy" e can be evaluated as :

$$
e=\varepsilon^{2}\left(\frac{1}{2}\left(\frac{D b}{D t}\right)^{2}-\frac{1}{2} \frac{D b}{D t} U_{x} h+\frac{U_{x}^{2} h^{2}}{6}\right)=\frac{\varepsilon^{2}}{2}\left(\left(\frac{D b}{D t}-\frac{U_{x} h}{2}\right)^{2}+\frac{U_{x}^{2} h^{2}}{12}\right)
$$

For the potential flows, one has $[1,9]$ :

$$
\int_{b}^{b+h} u^{2} d z \approx h U^{2}+O\left(\varepsilon^{4}\right)
$$

We will prove below a more general estimate covering also the case of weakly sheared flows. The flow is weakly sheared if in dimensionless variables one has :

$$
\frac{\partial u}{\partial z}=O\left(\varepsilon^{\alpha}\right), \quad \alpha>0 .
$$

This property is time invariant : if, initially, the flow is weakly sheared, then for any time it will also be weakly sheared. Indeed, in the long wave approximation the dimensionless vorticity is :

$$
\omega=-\frac{\partial u}{\partial z}+O\left(\varepsilon^{2}\right)
$$

Since in 2D case the vorticity is conserved along trajectories, one obtains :

$$
\left(\frac{\partial}{\partial t}+u \frac{\partial}{\partial x}+w \frac{\partial}{\partial z}\right) \frac{\partial u}{\partial z}=O\left(\varepsilon^{2}\right)
$$

It implies that for any time

$$
\frac{\partial u}{\partial z}=O(\beta), \quad \beta=\min (\alpha, 2)
$$

In particular, it implies that

$$
\frac{1}{h} \int_{b}^{b+h}|u-U|^{2} d z=O\left(\varepsilon^{2 \beta}\right) .
$$


Indeed, for any $z$ belonging to $(b, b+h)$ we have

$$
\begin{gathered}
|u-U|=\left|u-\frac{1}{h} \int_{b}^{b+h} u d z\right|=\left|\int_{b}^{z} \frac{\partial u}{\partial z} d z-\frac{1}{h} \int_{b}^{b+h}\left(\int_{b}^{z^{\prime}} \frac{\partial u}{\partial z} d z\right) d z^{\prime}\right| \\
\leq \int_{b}^{z}\left|\frac{\partial u}{\partial z}\right| d z+\frac{1}{h} \int_{b}^{b+h} \int_{b}^{z^{\prime}}\left|\frac{\partial u}{\partial z}\right| d z d z^{\prime} \leq \max _{b \leq z \leq b+h}\left|\frac{\partial u}{\partial z}\right|\left(\int_{b}^{b+h} d z+\frac{1}{h} \int_{b}^{b+h}\left(z^{\prime}-b\right) d z^{\prime}\right) \\
=\frac{3 h}{2} \max _{b \leq z \leq b+h}\left|\frac{\partial u}{\partial z}\right| .
\end{gathered}
$$

Hence,

$$
\frac{1}{h} \int_{b}^{b+h}|u-U|^{2} d z=O\left(\varepsilon^{2 \beta}\right) .
$$

Analogously, one can prove that

$$
\frac{1}{h} \int_{b}^{b+h}|u-U|^{3} d z=O\left(\varepsilon^{3 \beta}\right) .
$$

In particular, for the potential flows one has $\omega=0$ and $\beta=2$. Keeping only the terms of order $\varepsilon^{0}$ and $\varepsilon^{2}$ in (10), (11), (12) we obtain the final system for a lower potential layer with the entrainment at the interface $z=b+h$ :

$$
\begin{aligned}
& \frac{\partial h}{\partial t}+\frac{\partial}{\partial x}(h U)=-M \\
& \frac{\partial}{\partial t}(h U)+\frac{\partial}{\partial x}\left(h U^{2}+P\right)=\left.p\right|_{z=b+h}(b+h)_{x}-\left.p\right|_{z=b} b_{x}-\left.M u\right|_{z=b+h} \\
& \frac{\partial}{\partial t}\left(h\left(\frac{U^{2}}{2}+e+\frac{h}{2}+b\right)\right)+\frac{\partial}{\partial x}\left(h U\left(\frac{U^{2}}{2}+e+\frac{h}{2}+b\right)+U P\right) \\
& =\left.p\right|_{z=b} b_{t}-\left.p\right|_{z=b+h}\left(M+(b+h)_{t}\right) \\
& \quad-M\left(\left.\frac{u^{2}}{2}\right|_{z=b+h}+\frac{\varepsilon^{2}}{2}\left(\frac{D b}{D t}-U_{x} h\right)^{2}+b+h\right) .
\end{aligned}
$$

The value of velocity $\left.u\right|_{z=b+h}$ is not yet determined. Let us remark that we have three governing equations for only two unknowns $h$ and $U$. The compatibility condition between the energy, momentum and mass equations gives us only one possibility (for proof, see Appendix A):

$$
\left.u\right|_{z=b+h}=U \text {. }
$$

Such a closure was also used in [10]. Thus, only the pressure $p$ at the interface $z=b+h$ should be prescribed to close system (15). 
Since the fluid density is constant, it is sufficient to put $\varepsilon=1$ and "add" a multiplier $g$ in the pressure definition to return back to dimensional variables :

$$
\begin{aligned}
& \frac{\partial\left(\rho_{l} h\right)}{\partial t}+\frac{\partial\left(\rho_{l} U h\right)}{\partial x}=-M \rho_{l} \\
& \frac{\partial}{\partial t}\left(\rho_{l} h U\right)+\frac{\partial}{\partial x}\left(\rho_{l} h U^{2}+P\right)=\left.p\right|_{z=b+h}(b+h)_{x}-\left.p\right|_{z=b} b_{x}-M \rho_{l} U \\
& \frac{\partial G}{\partial t}+\frac{\partial}{\partial x}((G+P) U)=-\left.p\right|_{z=b+h}\left(M+(b+h)_{t}\right)+\left.p\right|_{z=b} b_{t} \\
& -M \rho_{l}\left(\frac{U^{2}}{2}+\frac{1}{2}\left(\frac{D b}{D t}-U_{x} h\right)^{2}+g(b+h)\right)
\end{aligned}
$$

where

$$
\begin{aligned}
& G=\rho_{l} h\left(\frac{U^{2}}{2}+e+\frac{g h}{2}+g b\right), \quad e=\frac{1}{2}\left(\left(\frac{D b}{D t}-\frac{U_{x} h}{2}\right)^{2}+\frac{U_{x}^{2} h^{2}}{12}\right), \\
& P=\left.p\right|_{z=b+h} h+\rho_{l}\left(\frac{g h^{2}}{2}+\left(\frac{h^{2}}{2} \frac{D^{2} b}{D t^{2}}-\frac{h^{3}}{3}\left(\frac{D U_{x}}{D t}-U_{x}^{2}\right)\right)\right) \\
& \left.p\right|_{z=b}=\left.p\right|_{z=b+h}+\rho_{l}\left(g h+h \frac{D^{2} b}{D t^{2}}-\frac{1}{2} h^{2}\left(\frac{D U_{x}}{D t}-U_{x}^{2}\right)\right) .
\end{aligned}
$$

\section{Subsurface bubble shear layer}

The upper layer of thickness $\eta(t, x)$ is situated in the domain $D_{t}=\{x, z \mid-\infty<x<$ $\infty, b+h<z<f\}$, where $z=f(t, x)=b(t, x)+h(t, x)+\eta(t, x)$ is the position of the free surface. This layer will be considered as a two-phase mixture of an incompressible gas (air) of density $\rho_{a}=$ const and incompressible fluid (water) of density $\rho_{l}=$ const (the same as in the lower layer). The equations are :

$$
\begin{aligned}
& \frac{\partial u}{\partial x}+\frac{\partial w}{\partial z}=0, \quad \frac{\partial \rho}{\partial t}+u \frac{\partial \rho}{\partial x}+w \frac{\partial \rho}{\partial z}=0 \\
& \rho\left(\frac{\partial u}{\partial t}+u \frac{\partial u}{\partial x}+w \frac{\partial u}{\partial z}\right)=-\frac{\partial p}{\partial x} \\
& \rho\left(\frac{\partial w}{\partial t}+u \frac{\partial w}{\partial x}+w \frac{\partial w}{\partial z}\right)=-\left(\rho g+\frac{\partial p}{\partial z}\right) .
\end{aligned}
$$

Here $\mathbf{u}=(u, w)^{T}$ is the mixture velocity, $\rho=\alpha_{a} \rho_{a}+\alpha_{l} \rho_{l}$ is the mixture density, $p=$ $\alpha_{a} p_{a}+\alpha_{l} p_{l}$ is the mixture pressure, $\alpha_{a}$ and $\alpha_{l}$ are the volume fractions of air and water, respectively, $\alpha_{a}+\alpha_{l}=1$. The two-layer system fluid is "stably stratified", because $\rho<\rho_{l}$ (or, equivalently, $\rho_{a}<\rho_{l}$ ). 
The conventional hydrostatic approximation of the governing equations for the upper layer is :

$$
\begin{aligned}
& \frac{\partial u}{\partial x}+\frac{\partial w}{\partial z}=0, \quad \frac{\partial \rho}{\partial t}+\frac{\partial(\rho u)}{\partial x}+\frac{\partial(\rho w)}{\partial z}=0 \\
& \frac{\partial(\rho u)}{\partial t}+\frac{\partial\left(\rho u^{2}+p\right)}{\partial x}+\frac{\partial(\rho u w)}{\partial z}=0, \quad p(t, x, z)=-g \int_{f}^{z} \rho(t, x, s) d s .
\end{aligned}
$$

This system is obtained by using the standard shallow water scaling (the terms of order $O\left(\varepsilon^{2}\right)$ are neglected in the corresponding dimensionless Euler equations of non-homogeneous fluids). The system admits the energy conservation law :

$$
\frac{\partial}{\partial t}\left(\rho\left(\frac{u^{2}}{2}+g z\right)\right)+\frac{\partial}{\partial x}\left(\left(\rho\left(\frac{u^{2}}{2}+g z\right)+p\right) u\right)+\frac{\partial}{\partial z}\left(\left(\rho\left(\frac{u^{2}}{2}+g z\right)+p\right) w\right)=0 .
$$

The boundary conditions are

$$
\frac{\partial f}{\partial t}+u \frac{\partial f}{\partial x}-\left.w\right|_{z=f}=0, \quad \frac{\partial(b+h)}{\partial t}+u \frac{\partial(b+h)}{\partial x}-\left.w\right|_{z=b+h}=-M .
$$

The first condition means that there is no bubble entrainment at the free surface (the bubble layer is already formed). The second condition describes the mixing between potential and shear layers (entrainment process).

For any function $F(t, x, z)$ we define its averaged value in the upper layer by :

$$
\bar{F}(t, x)=\frac{1}{\eta} \int_{b+h}^{f} F(t, x, s) d s .
$$

In particular, averaging the incompressibility and mass conservation laws we have:

$$
\frac{\partial \eta}{\partial t}+\frac{\partial(\eta \bar{u})}{\partial x}=M, \quad \frac{\partial(\eta \bar{\rho})}{\partial t}+\frac{\partial(\eta \overline{\rho u})}{\partial x}=\left.M \rho\right|_{z=b+h}
$$

The averaging procedure is quite standard. For example, integrating the incompressibility equation one has :

$$
\int_{b+h}^{f}\left(u_{x}+w_{z}\right) d z=\frac{\partial}{\partial x}\left(\int_{b+h}^{f} u d z\right)-\left.f_{x} u\right|_{z=f}+\left.(b+h)_{x} u\right|_{z=b+h}+\left.w\right|_{z=f}-\left.w\right|_{z=b+h}=0 .
$$

Using the kinematic boundary conditions, we obtain the first equation of (17). The analogous procedure was applied to obtain the second equation of (17).

The dynamic conditions at the free surface $z=f$ and interface $z=b+h$ were additionally used to obtain the averaged momentum equation :

$$
\frac{\partial}{\partial t}(\eta \overline{\rho u})+\frac{\partial}{\partial x}\left(\eta\left(\overline{\rho u^{2}}+\bar{p}\right)\right)=\left.M \rho u\right|_{z=b+h}-\left.p\right|_{z=b+h}(b+h)_{x}
$$


and the averaged energy equation :

$$
\begin{aligned}
& \frac{\partial}{\partial t}\left(\overline{\eta \rho\left(\frac{u^{2}}{2}+g z\right)}\right)+\frac{\partial}{\partial x}\left(\eta\left(\overline{\rho u\left(\frac{u^{2}}{2}+g z\right)}+\overline{p u}\right)\right)= \\
& =\left.M\left(\frac{\rho u^{2}}{2}+\rho g(b+h)\right)\right|_{z=b+h}+\left.p\right|_{z=b+h}\left(M+(b+h)_{t}\right) .
\end{aligned}
$$

As in the case of the equations for the lower layer, we need to estimate the correlations presented in the governing equations.

\subsection{Equation for vorticity}

We will derive first the equation for the vorticity vector $\boldsymbol{\omega}=$ curlu in the hydrostatic approximation. In the case of a "stratified" fluid, the estimates for the correlations become a little bit more complicated than in the case of homogeneous fluids. We present them below. In $2 \mathrm{D}$ case the vorticity has only one non-vanishing component (in $y$-direction). Hence, the general equation for the vorticity vector $\boldsymbol{\omega}$ :

$$
\left(\frac{\partial}{\partial t}+u \frac{\partial}{\partial x}+w \frac{\partial}{\partial z}\right)\left(\frac{\boldsymbol{\omega}}{\rho}\right)-\frac{\partial \mathbf{u}}{\partial \mathbf{x}}\left(\frac{\boldsymbol{\omega}}{\rho}\right)+\frac{\mathbf{g} \wedge \nabla \rho}{\rho^{2}}=0 .
$$

This can be simplified to :

$$
\left(\frac{\partial}{\partial t}+u \frac{\partial}{\partial x}+w \frac{\partial}{\partial z}\right)\left(\frac{\omega}{\rho}\right)=-\frac{g \rho_{x}}{\rho^{2}} .
$$

Here the vorticity is approximately given by $\omega \approx u_{z}$. The equation for $\rho_{z}=-\left(\rho_{l}-\rho_{a}\right)\left(\alpha_{a}\right)_{z}$ is :

$$
\left(\frac{\partial}{\partial t}+u \frac{\partial}{\partial x}+w \frac{\partial}{\partial z}\right)\left(\rho_{z}\right)=-u_{z} \rho_{x}-w_{z} \rho_{z}
$$

Using the incompressibility condition we rewrite finally the equation for $\rho_{z}$ as :

$$
\left(\frac{\partial}{\partial t}+u \frac{\partial}{\partial x}+w \frac{\partial}{\partial z}\right)\left(\rho_{z}\right)+\omega \rho_{x}-u_{x} \rho_{z}=0 .
$$

Equations (18) and (19) imply : if initially the gas volume fraction $\alpha_{a}$ is low

$$
\alpha_{a} \approx \delta, \quad 1 \gg \delta \gg \varepsilon^{2},
$$

and the velocity and the fraction gradients are small :

$$
\sqrt{\frac{H}{g}} u_{z}=O(\gamma), \quad H\left(\alpha_{a}\right)_{z}=O(\delta \gamma), \quad 1 \gg \gamma \gg \varepsilon^{2},
$$

then for any time

$$
\sqrt{\frac{H}{g}} u_{z}=O(\gamma+\delta), \quad H\left(\alpha_{a}\right)_{z}=O(\delta(\gamma+\delta) .
$$

As before, $H$ is a characteristic vertical scale. The proof is a consequence of the linearity of equations for $\omega$ and $\rho_{z}$ [31], and is reminiscent of that of section 2. We need now to estimate all correlations presented in the averaged equations. 


\subsection{Estimation of correlations}

Since

$$
|\rho-\bar{\rho}|=\left|\rho_{l}-\rho_{a}\right|\left|\alpha_{a}-\bar{\alpha}_{a}\right|=O(\delta(\gamma+\delta)), \quad|u-\bar{u}|=O(\gamma+\delta),
$$

we obtain the following asymptotic estimates of integrals :

$$
\begin{aligned}
& \int_{b+h}^{f} \rho u d z=\eta \overline{\rho u}=\eta \bar{\rho} \bar{u}+\int_{b+h}^{f}(u-\bar{u})(\rho-\bar{\rho}) d s=\eta \bar{\rho} \bar{u}+O\left(\delta(\gamma+\delta)^{2}\right), \\
& \int_{b+h}^{f} p(t, x, s) d s=g \bar{\rho} \frac{\eta^{2}}{2}-g \int_{b+h}^{f}\left(\int_{f}^{z}(\rho-\bar{\rho}) d s\right) d z=g \bar{\rho} \frac{\eta^{2}}{2}+O(\delta(\gamma+\delta)), \\
& \left.p\right|_{z=b+h}=-g \int_{f}^{b+h} \rho(t, x, s) d s=g \bar{\rho} \eta \\
& \int_{b+h}^{f} z \rho d z=\int_{b+h}^{f} z(\rho-\bar{\rho}) d z+\frac{\bar{\rho}}{2}\left(f^{2}-(b+h)^{2}\right)=\int_{b+h}^{f} z(\rho-\bar{\rho}) d z \\
& +\frac{\bar{\rho} \eta}{2}(f+b+h)=\frac{\bar{\rho}}{2} \eta(\eta+2(b+h))+O(\delta(\gamma+\delta)), \\
& \int_{b+h}^{f} \rho u^{2} d z=\eta \overline{\rho u^{2}}=\eta\left(\bar{\rho} \bar{u}^{2}+\bar{\rho} \overline{(u-\bar{u})^{2}}+\overline{(\rho-\bar{\rho})(u-\bar{u})^{2}}+2 \bar{u} \overline{(\rho-\bar{\rho})(u-\bar{u})}\right) \\
& =\eta\left(\bar{\rho} \bar{u}^{2}+\bar{\rho} \overline{(u-\bar{u})^{2}}+O\left(\delta(\gamma+\delta)^{3}+\delta(\gamma+\delta)^{2}\right)\right) .
\end{aligned}
$$

Since

$$
\begin{gathered}
\int_{b+h}^{f} u p d s=\bar{u} \int_{b+h}^{f} p d s+\int_{b+h}^{f}(u-\bar{u}) p d s=\bar{u} \int_{b+h}^{f} p d s \\
-g \int_{b+h}^{f}\left((u-\bar{u}) \int_{f(t, x)}^{z}(\rho-\bar{\rho}) d s\right) d z-g \int_{b+h}^{f}\left((u-\bar{u}) \int_{f(t, x)}^{z} \bar{\rho} d s\right) d z \\
=\bar{u} \int_{b+h}^{f} p d s-g \int_{b+h}^{f}\left((u-\bar{u}) \int_{f(t, x)}^{z}(\rho-\bar{\rho}) d s\right) d z-g \bar{\rho} \int_{b+h}^{f}(u-\bar{u})(z-f) d z,
\end{gathered}
$$

and

$$
\begin{aligned}
g \int_{b+h}^{f} z u \rho d z & =g \int_{b+h}^{f} z(u-\bar{u})(\rho-\bar{\rho}) d z+g \bar{\rho} \int_{b+h}^{f} z(u-\bar{u}) d z \\
& +g \bar{u} \int_{b+h}^{f} z(\rho-\bar{\rho}) d z+g \bar{u} \bar{\rho} \int_{b+h}^{f} z d z
\end{aligned}
$$


then

$$
\begin{gathered}
\int_{b+h}^{f} u p d s+g \int_{b+h}^{f} z u \rho d z=\bar{u} \int_{b+h}^{f} p d s-g \int_{b+h}^{f}\left((u-\bar{u}) \int_{f}^{z}(\rho-\bar{\rho}) d s\right) d z \\
\quad+g \int_{b+h}^{f} z(u-\bar{u})(\rho-\bar{\rho}) d z+g \bar{u} \int_{b+h}^{f} z(\rho-\bar{\rho}) d z+g \bar{u} \bar{\rho} \int_{b+h}^{f} z d z \\
=\bar{u} \int_{b+h}^{f} p d s+O\left(\delta(\delta+\gamma)^{2}+\delta(\delta+\gamma)\right)+g \bar{u} \bar{\rho} \frac{\eta}{2}(\eta+2(b+h)) \\
=g \bar{u} \bar{\rho}\left(\frac{\eta^{2}}{2}+\frac{\eta}{2}(\eta+2(b+h))\right)+O\left(\delta(\delta+\gamma)^{2}+\delta(\delta+\gamma)\right)
\end{gathered}
$$

Finally,

$$
\begin{gathered}
\int_{b+h}^{f} \rho u^{3} d z=\int_{b+h}^{f}(\rho-\bar{\rho})\left((u-\bar{u})^{3}+3(u-\bar{u})^{2} \bar{u}+3(u-\bar{u}) \bar{u}^{2}+\bar{u}^{3}\right) d z \\
=\eta \bar{\rho} \bar{u}^{3}+3 \bar{\rho} \bar{u} \int_{b+h}^{f}(u-\bar{u})^{2} d z \\
+O\left(\delta(\delta+\gamma)^{2}+\delta(\delta+\gamma)^{3}+\delta(\delta+\gamma)^{4}+(\delta+\gamma)^{3}\right) .
\end{gathered}
$$

We will use these estimates to simplify the averaged equations in the limit where the shear effects defined by the dimensionless parameter $\gamma$ (see the definition in (21)) are more important than the density variation defined by $\delta$ (see the definition in (20) :

$$
\gamma \gg \delta
$$

Keeping only the terms of order $\gamma^{2}$ we obtain the final equations written below already in dimensional form :

$$
\begin{gathered}
\frac{\partial \eta}{\partial t}+\frac{\partial \eta \bar{u}}{\partial x}=M, \quad \frac{\partial \bar{\rho} \eta}{\partial t}+\frac{\partial \bar{\rho} \eta \bar{u}}{\partial x}=\left.M \rho\right|_{z=b+h} \\
\frac{\partial}{\partial t}(\bar{\rho} \eta \bar{u})+\frac{\partial}{\partial x}\left(\bar{\rho} \eta\left(\bar{u}^{2}+q^{2}\right)+g \bar{\rho} \frac{\eta^{2}}{2}\right)=\left.M \rho u\right|_{z=b+h}-\left.p\right|_{z=b+h}(b+h)_{x} \\
\frac{\partial}{\partial t}\left(\bar{\rho} \eta\left(\frac{\bar{u}^{2}}{2}+\frac{q^{2}}{2}+\frac{g}{2}(\eta+2(b+h))\right)\right) \\
+\frac{\partial}{\partial x}\left(\bar{\rho} \eta \bar{u}\left(\frac{\bar{u}^{2}}{2}+\frac{q^{2}}{2}+\frac{g}{2}(\eta+2(b+h))\right)+\bar{\rho} \bar{u}\left(\eta q^{2}+g \frac{\eta^{2}}{2}\right)\right) \\
=\left.M\left(\frac{\rho u^{2}}{2}+\rho g(b+h)\right)\right|_{z=b+h}+\left.p\right|_{z=b+h}\left(M+(b+h)_{t}\right) .
\end{gathered}
$$

Here the variable $q$ measuring the distortion of the velocity profile is defined as :

$$
q^{2}=\overline{(u-\bar{u})^{2}}
$$


The following boundary conditions should also be added:

$$
\left.p\right|_{z=b+h}=g \bar{\rho} \eta,\left.\quad \rho\right|_{z=b+h}=\rho_{l},\left.\quad u\right|_{z=b+h}=U .
$$

The first relation is just the definition of the hydrostatic pressure, the second relation comes from the continuity of density at the boundary of mixing layer, and the last relation comes from the compatibility with the energy equation (for proof, see Appendix A). The entrainment velocity $M$ is taken in the form $[10,16,17]$ :

$$
M=\sigma q, \quad \sigma=\text { const }>0 .
$$

The last step will be the following modification of the energy equation for the subsurface layer :

$$
\begin{gathered}
\frac{\partial}{\partial t}\left(\bar{\rho} \eta\left(\frac{\bar{u}^{2}}{2}+\frac{q^{2}}{2}+\frac{g}{2}(\eta+2(b+h))\right)\right) \\
+\frac{\partial}{\partial x}\left(\bar{\rho} \eta \bar{u}\left(\frac{\bar{u}^{2}}{2}+\frac{3}{2} q^{2}+g(b+h+\eta)\right)\right) \\
=M\left(\frac{\rho_{l} U^{2}}{2}+\rho_{l} g(b+h)\right)+g \bar{\rho} \eta\left(M+(b+h)_{t}\right)-\bar{\rho} d+M \rho_{l} \frac{\left.w^{2}\right|_{z=b+h}}{2} .
\end{gathered}
$$

Here the vertical velocity $w$ at the boundary $z=b+h$ comes from the free divergence condition :

$$
\left.w\right|_{z=b+h}=b_{t}+U b_{x}-h U_{x}=\frac{D b}{D t}-h U_{x},
$$

Two extra terms have been added into the equation of energy (25). The first one defined as

$$
\bar{\rho} d=\bar{\rho} \frac{\sigma \kappa}{2}|q|^{3}>0, \kappa>0,
$$

is responsible for the energy dissipation from large vortexes to small vortexes. The factor $1 / 2$ is added for convenience. This term was already introduced in [10].

The second term is added to have the compatibility with the full energy equation. Indeed, the equations in the upper shear layer are obtained in lower order with respect to $\varepsilon^{2}$, while the equations for the lower layer are obtained up to order $\varepsilon^{2}$. Thus, this term assures the energy conservation, when the term $d$ is absent. In particular, this guarantees that the energy can not increase. In applications, this term is almost vanishing.

\subsection{Equation for the distortion variable $q$}

We will derive here an equation for the variable $q$ defined by (23) and measuring the distortion of the velocity profile. Let us introduce

$$
\pi=\bar{\rho} \eta q^{2}+\frac{g}{2} \bar{\rho} \eta^{2} .
$$

Then the momentum equation is :

$$
\frac{\partial}{\partial t}(\bar{\rho} \eta \bar{u})+\frac{\partial}{\partial x}\left(\bar{\rho} \eta \bar{u}^{2}+\pi\right)=M \rho_{l} U-g \bar{\rho} \eta(b+h)_{x} .
$$


Its non-conservative form is :

$$
\bar{\rho} \eta \frac{D \bar{u}}{D t}+\pi_{x}=M \rho_{l}(U-\bar{u})-g \bar{\rho} \eta(b+h)_{x}
$$

The non-conservative form $(25)$ is :

$$
\begin{gathered}
\bar{\rho} \eta \frac{D}{D t}\left(\frac{\bar{u}^{2}}{2}+\frac{q^{2}}{2}+\frac{g}{2}(\eta+2(b+h))\right)+\left(\frac{\bar{u}^{2}}{2}+\frac{q^{2}}{2}+\frac{g}{2}(\eta+2(b+h))\right) M \rho_{l} \\
+\pi_{x} \bar{u}+\left(\bar{\rho} \eta q^{2}+\frac{g}{2} \bar{\rho} \eta^{2}\right) \bar{u}_{x}=M\left(\frac{\rho_{l} U^{2}}{2}+\rho_{l} g(b+h)\right)+g \bar{\rho} \eta\left(M+(b+h)_{t}\right) \\
-\bar{\rho} d+M \rho_{l} \frac{w^{2}}{2}
\end{gathered}
$$

It gives :

$$
\begin{gathered}
\bar{u}\left(-\pi_{x}+M \rho_{l}(U-\bar{u})-g \bar{\rho} \eta(b+h)_{x}\right) \\
+\bar{\rho} \eta \frac{D}{D t}\left(\frac{q^{2}}{2}+\frac{g}{2}(\eta+2(b+h))\right)+\left(\frac{\bar{u}^{2}}{2}+\frac{q^{2}}{2}+\frac{g}{2}(\eta+2(b+h))\right) M \rho_{l} \\
+\pi_{x} \bar{u}+\left(\bar{\rho} \eta q^{2}+\frac{g}{2} \bar{\rho} \eta^{2}\right) \bar{u}_{x}=M\left(\frac{\rho_{l} U^{2}}{2}+\rho_{l} g(b+h)\right)+g \bar{\rho} \eta\left(M+(b+h)_{t}\right) \\
-\bar{\rho} d+M \rho_{l} \frac{w^{2}}{2}
\end{gathered}
$$

Or :

$$
\begin{gathered}
\bar{u} M \rho_{l}(U-\bar{u}) \\
+\bar{\rho} \eta \frac{D}{D t}\left(\frac{q^{2}}{2}+\frac{g}{2} \eta\right)+\left(\frac{\bar{u}^{2}}{2}+\frac{q^{2}}{2}+\frac{g}{2}(\eta+2(b+h))\right) M \rho_{l} \\
+\left(\bar{\rho} \eta q^{2}+\frac{g}{2} \bar{\rho} \eta^{2}\right) \bar{u}_{x}=M\left(\frac{\rho_{l} U^{2}}{2}+\rho_{l} g(b+h)\right)+g \bar{\rho} \eta M-\bar{\rho} d+M \rho_{l} \frac{w^{2}}{2} .
\end{gathered}
$$

It is equivalent to :

$$
\begin{aligned}
& \bar{u} M \rho_{l}(U-\bar{u})+\bar{\rho} \eta \frac{D}{D t}\left(\frac{q^{2}}{2}\right)+\left(\frac{\bar{u}^{2}}{2}+\frac{q^{2}}{2}+\frac{g}{2} \eta\right) M \rho_{l} \\
& +\left(\bar{\rho} \eta q^{2}\right) \bar{u}_{x}=M\left(\frac{\rho_{l} U^{2}}{2}\right)+\frac{g}{2} M g \bar{\rho} \eta-\bar{\rho} d+M \rho_{l} \frac{w^{2}}{2} .
\end{aligned}
$$

Finally, the equation for $q$ is :

$$
q_{t}+(q \bar{u})_{x}=\frac{M \rho_{l}}{2 \bar{\rho} \eta q}\left((U-\bar{u})^{2}+w^{2}-q^{2}-\frac{g \eta\left(\rho_{l}-\bar{\rho}\right)}{\rho_{l}}\right)-\frac{d}{\eta q},
$$

where $w$ is given by (26). From the evolution equation (27) and the mass equation for $\eta$ one can derive the transport equation for $q / \eta$ which can be interpreted as the evolution equation for the flow vorticity in the upper layer. A priori, the vorticity can change its sign during evolution. 


\section{Mild slope approximation}

The equations can considerably be simplified under the following assumption. Let the dimensionless bottom variation be weak: $b=b\left(\varepsilon^{\alpha} t, \varepsilon^{\alpha} x\right), \alpha>0$. Then one can obviously neglect the terms containing the squared gradients and second-order derivatives of $b$, and keep on the right-hand side of the dimensionless momentum equation for the upper layer only the first gradient of $b$. Then the dimensional equations for the upper layer are :

$$
\begin{gathered}
\frac{\partial \eta}{\partial t}+\frac{\partial \eta \bar{u}}{\partial x}=M, \quad \frac{\partial \bar{\rho} \eta}{\partial t}+\frac{\partial \bar{\rho} \eta \bar{u}}{\partial x}=M \rho_{l}, \\
\frac{\partial}{\partial t}(\bar{\rho} \eta \bar{u})+\frac{\partial}{\partial x}\left(\bar{\rho} \eta\left(\bar{u}^{2}+q^{2}\right)+g \bar{\rho} \frac{\eta^{2}}{2}\right)=M \rho_{l} U-g \bar{\rho} \eta(b+h)_{x} \\
\frac{\partial}{\partial t}\left(\bar{\rho} \eta\left(\frac{\bar{u}^{2}}{2}+\frac{q^{2}}{2}+\frac{g}{2}(\eta+2(b+h))\right)\right) \\
+\frac{\partial}{\partial x}\left(\bar{\rho} \eta \bar{u}\left(\frac{\bar{u}^{2}}{2}+\frac{q^{2}}{2}+\frac{g}{2}(\eta+2(b+h))\right)+\bar{\rho} \bar{u} \eta\left(q^{2}+g \frac{\eta}{2}\right)\right) \\
=M \rho_{l}\left(\frac{U^{2}}{2}+g(b+h)\right)+g \bar{\rho} \eta\left(M+(b+h)_{t}\right)-\bar{\rho} d+\beta M \rho_{l} \frac{w^{2}}{2},
\end{gathered}
$$

where

$$
M=\sigma q, \quad d=\frac{\sigma \kappa}{2}|q|^{3}, \quad w=-U_{x} h .
$$

The parameter $\beta=0(\beta=1)$ corresponds to the hydrostatic (non-hydrostatic) approximation of the governing equations.

The dimensional equations for the lower layer in a mild slope approximation are :

$$
\begin{gathered}
\frac{\partial \rho_{l} h}{\partial t}+\frac{\partial\left(\rho_{l} U h\right)}{\partial x}=-M \rho_{l}, \\
\frac{\partial}{\partial t}\left(\rho_{l} h U\right)+\frac{\partial}{\partial x}\left(\rho_{l} h U^{2}+P\right)=\bar{\rho} g \eta(b+h)_{x}-\left.p\right|_{z=b} b_{x}-M \rho_{l} U .
\end{gathered}
$$

Here

$$
\begin{aligned}
& P=g \bar{\rho} \eta h+\rho_{l}\left(\frac{g h^{2}}{2}-\frac{\beta}{3} h^{3}\left(\frac{D U_{x}}{D t}-U_{x}^{2}\right)\right), \\
& \left.p\right|_{z=b}=g \bar{\rho} \eta+\rho_{l}\left(g h-\frac{\beta}{2} h^{2}\left(\frac{D U_{x}}{D t}-U_{x}^{2}\right)\right),
\end{aligned}
$$

Equations (28)-(32) form a closed system for six unknown functions $\eta, \bar{\rho}, \bar{u}, q, h$, and $U$. The energy equation

$$
\begin{gathered}
\frac{\partial G}{\partial t}+\frac{\partial}{\partial x}((G+P) U)=-g \bar{\rho} \eta\left(M+(b+h)_{t}\right)+\left.p\right|_{z=b} b_{t} \\
-M \rho_{l}\left(\frac{U^{2}}{2}+\frac{\beta}{2} w^{2}+g(b+h)\right)
\end{gathered}
$$


is a consequence of equations (31) and (32). Here

$$
G=\rho_{l} h\left(\frac{U^{2}}{2}+e+\frac{g h}{2}+g b\right), \quad e=\frac{\beta}{6} U_{x}^{2} h^{2} .
$$

The full energy equation is obtained by adding (30) and (33).

\section{Exact solutions}

In this section we present exact periodic solutions to our equations.

\subsection{Time periodic solutions}

Consider now oscillations of bubble clouds over a flat bottom $(b=0)$ that are homogeneous in space (the dependence on the space coordinate $x$ is absent). We also neglect the dissipation effects $(\kappa=0)$.

The governing equations (28)-(33) are :

$$
\begin{aligned}
& \frac{\partial h}{\partial t}=-\sigma q, \quad \frac{\partial \eta}{\partial t}=\sigma q, \quad \frac{\partial}{\partial t}\left(\eta\left(\bar{\rho}-\rho_{l}\right)\right)=0 \\
& \frac{\partial}{\partial t}\left(\bar{\rho} \eta \bar{u}+\rho_{l} h U\right)=0, \quad \frac{\partial U}{\partial t}=0 \\
& \frac{\partial}{\partial t}\left(\bar{\rho} \eta\left(\frac{\bar{u}^{2}}{2}+\frac{q^{2}}{2}+\frac{g}{2}(\eta+2 h)\right)+\rho_{l} h\left(\frac{U^{2}}{2}+\frac{g h}{2}\right)\right)=0
\end{aligned}
$$

Let us note that in this case the governing equations for the hydrostatic and non-hydrostatic approximations are the same. One can always suppose that $U=$ const $=0$. One has then :

$$
\begin{aligned}
& \frac{\partial h}{\partial t}=-\sigma q, \quad \frac{\partial \eta}{\partial t}=\sigma q, \quad \eta\left(\rho_{l}-\bar{\rho}\right)=\rho_{l} \mathcal{L}=\text { const }>0 \\
& \bar{\rho} \eta \bar{u}=\rho_{l} \mathcal{H} \mathcal{V}=\text { const }, \quad h+\eta=\mathcal{H}=\text { const } \\
& \bar{\rho} \eta\left(\frac{\bar{u}^{2}}{2}+\frac{q^{2}}{2}+\frac{g}{2}(\eta+2 h)\right)+\rho_{l} \frac{g h^{2}}{2}=\rho_{l} \mathcal{H} \mathcal{E}=\text { const. }
\end{aligned}
$$

Here the constants $\mathcal{L}, \mathcal{H}$ have the dimension of length, while $\mathcal{V}$ and $\mathcal{E}$ have the dimension of velocity and squared velocity, respectively. Eliminating $h$, one can transform the energy equation into the form :

$$
\bar{\rho} \eta\left(\frac{\bar{u}^{2}}{2}+\frac{q^{2}}{2}\right)+\frac{g}{2} \rho_{l} \mathcal{L} \eta=\rho_{l} \mathcal{H} \mathcal{E}-\rho_{l} \frac{g \mathcal{H}^{2}}{2}+g \rho_{l} \mathcal{L} \mathcal{H} .
$$

Finally, it implies :

$$
\frac{\mathcal{V}^{2} \mathcal{H}^{2}}{2(\eta-\mathcal{L})^{2}}+\frac{q^{2}}{2}+\frac{g \mathcal{L}}{2} \frac{\eta}{\eta-\mathcal{L}}=\frac{\mathcal{B}}{\eta-\mathcal{L}}, \quad \mathcal{B}=\mathcal{H}(\mathcal{E}-g \mathcal{H} / 2+g \mathcal{L})>0
$$




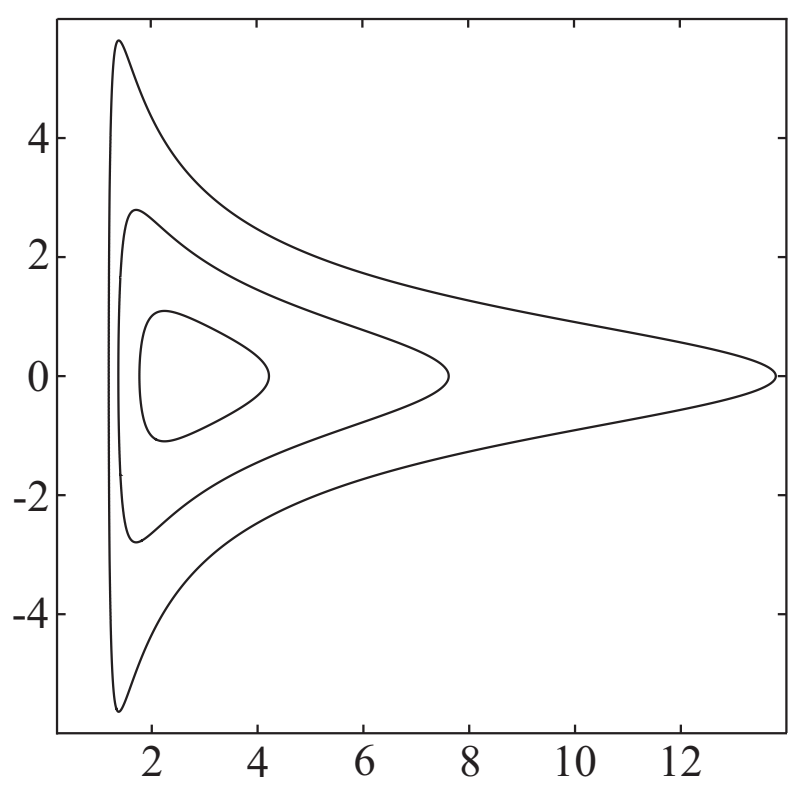

Figure 2: Level sets of the function $(34)$ is shown in the $(\eta, q)$ - plane for different parameters $\mathcal{B}$. The closed curves correspond to periodic solutions.

Together with the equation $\eta_{t}=\sigma q$ they form a closed system of equations.

Let us denote :

$$
W(\eta)=\frac{\mathcal{V}^{2} \mathcal{H}^{2}}{2(\eta-\mathcal{L})^{2}}+\frac{g \mathcal{L}}{2} \frac{\eta}{\eta-\mathcal{L}}-\frac{\mathcal{B}}{\eta-\mathcal{L}} .
$$

One has :

$$
\begin{aligned}
\frac{d W}{d \eta} & =-\frac{\mathcal{V}^{2} \mathcal{H}^{2}}{(\eta-\mathcal{L})^{3}}-\frac{g \mathcal{L}^{2}}{2} \frac{1}{(\eta-\mathcal{L})^{2}}+\frac{\mathcal{B}}{(\eta-\mathcal{L})^{2}} \\
\frac{d^{2} W}{d \eta^{2}} & =\frac{3 \mathcal{V}^{2} \mathcal{H}^{2}}{(\eta-\mathcal{L})^{4}}+\frac{g \mathcal{L}^{2}}{(\eta-\mathcal{L})^{3}}-\frac{2 \mathcal{B}}{(\eta-\mathcal{L})^{3}} .
\end{aligned}
$$

At the extremum point $\eta_{c}$ where $W^{\prime}\left(\eta_{c}\right)=0$ one has :

$$
\begin{gathered}
W\left(\eta_{c}\right)=\frac{g \mathcal{L}}{2}-\frac{\mathcal{V}^{2} \mathcal{H}^{2}}{2(\eta-\mathcal{L})^{2}}, \quad \frac{d^{2} W}{d \eta^{2}}\left(\eta_{c}\right)=\frac{3 \mathcal{V}^{2} \mathcal{H}^{2}}{(\eta-\mathcal{L})^{4}}+\frac{g \mathcal{L}^{2}}{(\eta-\mathcal{L})^{3}}- \\
\frac{2}{\eta-\mathcal{L}}\left(\frac{\mathcal{V}^{2} \mathcal{H}^{2}}{(\eta-\mathcal{L})^{3}}+\frac{g \mathcal{L}^{2}}{2} \frac{1}{(\eta-\mathcal{L})^{2}}\right)=\frac{\mathcal{V}^{2} \mathcal{H}^{2}}{(\eta-\mathcal{L})^{4}}>0 .
\end{gathered}
$$

Hence, this is a minimum point. A typical level set structure of the function (34) is shown in Fig. 2. In the case where $W\left(\eta_{c}\right)<0$, this corresponds to the time periodic solutions to our system.

\subsection{Periodic stationary waves}

We study here stationary solutions to (28)-(33) over flat bottom for both hydrostatic $(\beta=0)$ and non-hydrostatic $(\beta=1)$ cases. The stationary solutions to the non-hydrostatic 
model written in dimensional form satisfy the equations:

$$
\begin{aligned}
& (U h)^{\prime}=-\sigma q, \quad(\bar{u} \eta)^{\prime}=\sigma q, \quad(\bar{u} \bar{\rho} \eta)^{\prime}=\sigma q \rho_{l}, \\
& \left(\left(\bar{u}^{2}+q^{2}\right) \bar{\rho} \eta+\frac{g \bar{\rho} \eta^{2}}{2}\right)^{\prime}=\sigma q \rho_{l} U-g \bar{\rho} \eta h^{\prime}, \quad(\bar{u} q)^{\prime}=F \\
& \left(\frac{U^{2}}{2}-h U h^{\prime} U^{\prime}-\frac{1}{3} h^{2} U U^{\prime \prime}+g \eta \frac{\bar{\rho}}{\rho_{l}}-\frac{1}{2} h^{2} U^{\prime 2}-\sigma q h U^{\prime}\right)^{\prime}=-g h^{\prime}-\frac{\sigma q}{3} h U^{\prime \prime},
\end{aligned}
$$

where

$$
F=\frac{\sigma \rho_{l}}{2 \bar{\rho} \eta}\left((U-\bar{u})^{2}+\left(h U^{\prime}\right)^{2}-\left(1+\frac{\kappa \bar{\rho}}{\rho_{l}} \operatorname{sgn}(q)\right) q^{2}-\left(1-\frac{\bar{\rho}}{\rho_{l}}\right) g \eta\right) .
$$

Here we denote by 'prime' the derivative with respect to $x$. The normal form of (35) is :

$$
\begin{aligned}
& U^{\prime}=r \quad r^{\prime}=s, \quad h^{\prime}=-\frac{\sigma q+h r}{U}, \quad \bar{\rho}^{\prime}=\frac{\sigma q}{\bar{u} \eta}, \\
& \bar{u}^{\prime}=\frac{G}{\Delta}, \quad q^{\prime}=\frac{F-q \bar{u}^{\prime}}{\bar{u}}, \quad \eta^{\prime}=\frac{\sigma q-\eta \bar{u}^{\prime}}{\bar{u}}, \\
& s^{\prime}=\frac{3}{U h^{2}}\left(U r+g h^{\prime}+\frac{g \eta}{\rho_{l}} \bar{\rho}^{\prime}+\frac{g \bar{\rho}}{\rho_{l}} \eta^{\prime}+\frac{1}{3} h^{2} r s-\left(U s-r^{2}\right) h h^{\prime}\right),
\end{aligned}
$$

where

$$
\begin{aligned}
G & =\frac{\sigma q \rho_{l}}{\bar{\rho} \eta}\left(U \bar{u}-\left(\bar{u}^{2}+q^{2}+g \eta / 2\right)\right)-2 q F-\frac{g \sigma q}{2}-g \bar{u} h^{\prime}, \\
\Delta & =\bar{u}^{2}-3 q^{2}-g \eta .
\end{aligned}
$$

The 'true' normal form is obtained by replacing the derivatives appearing on the right hand side by the corresponding algebraic relations.

The stationary solutions to the hydrostatic model satisfy the following system of ODEs :

$$
\begin{aligned}
& h^{\prime}=-\frac{g h}{U^{2}-g h}\left(\frac{\bar{\rho} \eta}{\rho_{l} \bar{u}} \bar{u}^{\prime}+\left(\frac{U}{g h}-\frac{1}{\bar{u}}\right) \sigma q\right), \quad \eta^{\prime}=\frac{\sigma q-\eta \bar{u}^{\prime}}{\bar{u}}, \\
& \bar{\rho}^{\prime}=\frac{\sigma q}{\bar{u} \eta}\left(\rho_{l}-\bar{\rho}\right), \quad \bar{u}^{\prime}=\frac{G_{1}}{\Delta_{1}}, \quad q^{\prime}=\frac{F_{1}-q \bar{u}^{\prime}}{\bar{u}}, \quad U^{\prime}=-\frac{\sigma q+U h^{\prime}}{h},
\end{aligned}
$$

where

$$
\begin{aligned}
& F_{1}=\frac{\sigma \rho_{l}}{2 \bar{\rho} \eta}\left((U-\bar{u})^{2}-\left(1+\frac{\kappa \bar{\rho}}{\rho_{l}} \operatorname{sgn}(q)\right) q^{2}-\left(1-\frac{\bar{\rho}}{\rho_{l}}\right) g \eta\right), \\
& G_{1}=\frac{g \sigma q}{U^{2}-g h}(U \bar{u}-g h)+\frac{\sigma q \rho_{l}}{\bar{\rho} \eta}-2 q F_{1}-\frac{g \sigma q}{2} \\
& \Delta_{1}=\bar{u}^{2}-3 q^{2}-g \eta\left(1+\frac{g h \bar{\rho}}{\left(U^{2}-g h\right) \rho_{l}}\right) .
\end{aligned}
$$


In the case $\kappa=0$ one can find five first integrals of $(37)$ :

$$
\begin{aligned}
& U h+\bar{u} \eta=Q_{0}, \quad \rho_{l} U h+\bar{\rho} \bar{u} \eta=M_{0}, \quad \frac{1}{2} U^{2}+g h+g \eta \frac{\bar{\rho}}{\rho_{l}}=J_{0}, \\
& U^{2} h+\left(\bar{u}^{2}+q^{2}\right) \eta \frac{\bar{\rho}}{\rho_{l}}+\frac{g h^{2}}{2}+g h \eta \frac{\bar{\rho}}{\rho_{l}}+\frac{g \eta^{2}}{2} \frac{\bar{\rho}}{\rho_{l}}=K_{0}, \\
& U^{3} h+\left(\bar{u}^{2}+3 q^{2}\right) \bar{u} \eta \frac{\bar{\rho}}{\rho_{l}}+2 g\left(U h\left(h+\eta \frac{\bar{\rho}}{\rho_{l}}\right)+\bar{u} \eta(h+\eta) \frac{\bar{\rho}}{\rho_{l}}\right)=E_{0} .
\end{aligned}
$$

Here $Q_{0}, M_{0}, J_{0}, K_{0}$, and $E_{0}$ are constants. These integrals allow us to rigorously prove the existence of periodic solutions of (37).

Let us introduce the variable $Q=\bar{u} \eta$. Using the first four integrals of (38) one can present $h, \bar{\rho}, \eta, \bar{u}$, and $q^{2}$ as functions of variables $Q$ and $U$ :

$$
\begin{aligned}
& h=\frac{Q_{0}-Q}{U}, \quad \bar{\rho}=\frac{M_{0}-\left(Q-Q_{0}\right) \rho_{l}}{Q}, \quad \eta=\frac{\rho_{l}}{g \bar{\rho}}\left(J_{0}-\frac{U^{2}}{2}-g h\right), \\
& \bar{u}=\frac{Q}{\eta}, \quad q^{2}=\frac{\rho_{l}}{\bar{\rho} \eta}\left(K_{0}-\left(Q_{0}-Q\right) U-\frac{g h^{2}}{2}\right)-\frac{Q^{2}}{\eta^{2}}-\frac{g \eta}{2}-g h .
\end{aligned}
$$

Substituting the previous expressions into the last integral of (38), one obtains an algebraic equation $W(Q, U)=E_{0}$. One can prove that $\frac{d W}{d Q} \neq 0$. This allows us to determine (implicitly) the dependence $Q=Q(U)$. Thus, all unknowns are represented as functions of variable $U$. In Fig. 3 the dependences $Q(U)$ and $q^{2}(U)$ (multiplied, for a better visibility, by the scale factor 50) are shown for the following values of $Q_{0}, M_{0}, J_{0}, K_{0}$, and $E_{0}$ (taken at $x=0)$ :

$$
h_{0}=1, \quad \eta_{0}=0.1, \quad U_{0}=0.4, \quad \bar{\rho}_{0}=0.9, \quad \bar{u}_{0}=0.54, \quad q_{0}=0 .
$$

We also take $g=1$ and $\sigma=0.15$. As we can see from the Fig. 3 , the variable $q^{2}(U)$ vanishes at $U_{0}=0.4$ and $U_{m} \approx 0.39935$. This fact allows us to construct the periodic solutions.

For this, we introduce the functions $q=q^{ \pm}= \pm \sqrt{q^{2}(U)}$. Taking into account that $d Q / d x=\sigma q$, the dependence of the solution on $x$ is given implicitly by the equation :

$$
x=-\int_{U}^{U_{0}} \frac{Q^{\prime}(U) d U}{\sigma q^{+}(U)},
$$

when $U$ varies from $U_{0}$ to $U_{m}$. At the point $U=U_{m}$ one can 'switch' to the negative branch $q=q^{-}(U)$ and represent the solution in the form :

$$
x=X+\int_{U_{m}}^{U} \frac{Q^{\prime}(U) d U}{\sigma q^{-}(U)},
$$




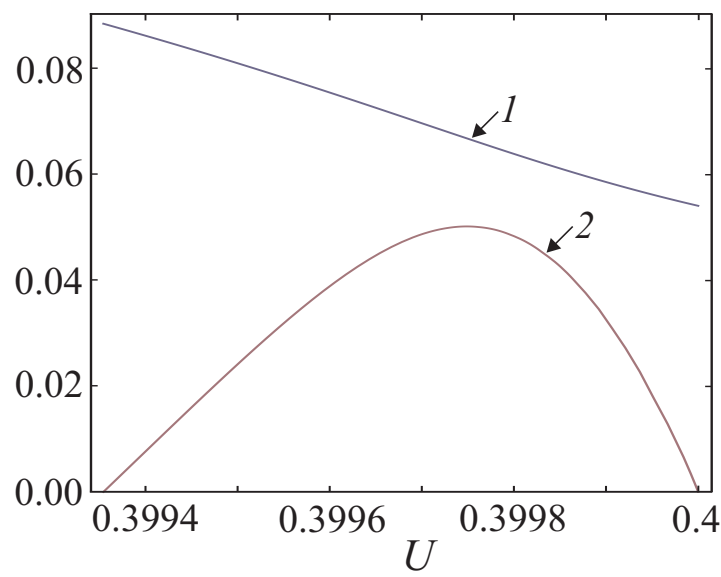

Figure 3: Functions $Q(U)$ (curve 1) and $q^{2}(U)$ with factor 50 (curve 2) obtained from integrals (38) for data (39).

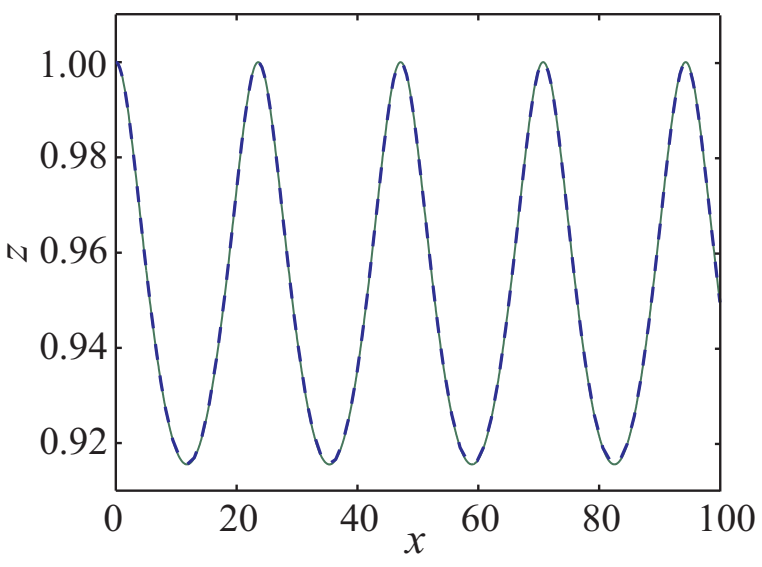

Figure 4: The solution $h(x)$ of equations (36) (solid line) and (37) (dashed line) for data (39).

where $U$ varies from $U_{m}$ to $U_{0}$. Here $X$ is the half-period of space oscillations, defined as :

$$
X=-\int_{U_{m}}^{U_{0}} \frac{Q^{\prime}(U) d U}{\sigma q^{+}(U)}=\int_{U_{m}}^{U_{0}} \frac{Q^{\prime}(U) d U}{\sigma q^{-}(U)} .
$$

We also note that the integral on the right-hand side of (41) (as well as of (40)) converges because $q^{ \pm}(U) \approx \pm \alpha_{1} \sqrt{U_{0}-U}$ and $q^{ \pm}(U) \approx \pm \alpha_{2} \sqrt{U-U_{m}}$ in the vicinity of the endpoints $U_{0}$ and $U_{m}$ (here $\alpha_{1}, \alpha_{2}$ are positive constants), and $Q^{\prime}(U)<0$ is a smooth function on $\left[U_{m}, U_{0}\right]$. Moreover, in this specific case $X \approx 11.7$. Thus, we have constructed a periodic solution for the hydrostatic system (37). In Fig. 4 the depth of the lower layer $h(x)$ is shown (dashed line). The maximum (minimal) value of $h$ is $h=1(h \approx 0.915)$ is attained at the points $x=2 n X(x=(2 n+1) X, n$ is integer $)$, and corresponds to $U=U_{0}\left(U=U_{m}\right)$.

In the case $\kappa=0$ and $\sigma=0.15$, the non-hydrostatic (36) and hydrostatic (37) equations were numerically solved by using the Runge-Kutta methods. The interface $z=h$ corresponding to both periodic solutions is shown in Fig. 4 for the 'initial' conditions (39) at $x=0$. The solid line corresponds to the non-hydrostatic model (36), while the dashed line corresponds to the hydrostatic model (37). We note that for the parameters (39) there is no difference in numerical results obtained in the framework of hydrostatic and non-hydrostatic models. 


\section{Non-stationary flows over obstacle}

\subsection{Numerical method}

For a numerical treatment of non-stationary problems, the following form of the governing equations (28)-(33) is used:

$$
\begin{aligned}
& \frac{\partial h}{\partial t}+\frac{\partial}{\partial x}(h U)=-\sigma q, \quad \frac{\partial \eta}{\partial t}+\frac{\partial}{\partial x}(\eta \bar{u})=\sigma q, \quad \frac{\partial}{\partial t}(\bar{\rho} \eta)+\frac{\partial}{\partial x}(\bar{u} \bar{\rho} \eta)=\sigma q \rho_{l}, \\
& \frac{\partial}{\partial t}(\bar{u} \bar{\rho} \eta)+\frac{\partial}{\partial x}\left(\left(\bar{u}^{2}+q^{2}\right) \bar{\rho} \eta+\frac{g \bar{\rho} \eta^{2}}{2}\right)=\sigma q \rho_{l} U-g \bar{\rho} \eta b_{x}+\alpha_{1}, \\
& \frac{\partial q}{\partial t}+\frac{\partial}{\partial x}(q \bar{u})=\frac{\sigma \rho_{l}}{2 \bar{\rho} \eta}\left((U-\bar{u})^{2}+w^{2}-\left(1+\frac{\kappa \bar{\rho}}{\rho_{l}} \operatorname{sgn}(q)\right) q^{2}-\left(1-\frac{\bar{\rho}}{\rho_{l}}\right) g \eta\right), \\
& \frac{\partial K}{\partial t}+\frac{\partial}{\partial x}\left(K U-\frac{U^{2}}{2}+g\left(h+\frac{\bar{\rho} \eta}{\rho_{l}}\right)+\alpha_{2}\right)=\sigma q\left(\frac{K-U}{h}+\alpha_{3}\right)-g b_{x},
\end{aligned}
$$

where

$$
\alpha_{1}=-g \bar{\rho} \eta h_{x}, \quad \alpha_{2}=-\frac{h^{2} U_{x}^{2}}{2}-\sigma q h U_{x}, \quad \alpha_{3}=h_{x} U_{x}, \quad w=-h U_{x},
$$

and the velocity $U$ is determined by the equation :

$$
K=U-\frac{1}{3 h}\left(h^{3} U_{x}\right)_{x}
$$

For given $K$ and $h$, equation (44) is an ordinary differential equation for $U$. The evolution equation for $K$ (the last relation in (42)) is equivalent to the momentum equation (for proof, see Appendix B).

The system (42) can be written in conservative form :

$$
\frac{\partial \mathbf{u}}{\partial t}+\frac{\partial \mathbf{f}}{\partial x}=\mathbf{g}
$$

where $\mathbf{u}=(h, \eta, \bar{\rho} \eta, \bar{u} \bar{\rho} \eta, q, K)^{\mathrm{T}}$ is the vector of unknowns, $\mathbf{f}$ and $\mathbf{g}$ are the corresponding flux and right-hand side.

Following [15], we divide the numerical resolution of system (42) into three successive steps:

1) numerical approximations of the terms $w$ and $\alpha_{i}, i=1,2,3$ containing spatial and time derivatives (see relations (43));

2) time evolution of the conservative variables $\mathbf{u}$ using Godunov-type method;

3) resolution of an ordinary differential equation (44) to find the velocity $U$.

To solve balance laws (45) numerically, we implemented the Nessyahu-Tadmor secondorder central scheme [23, 26]. The details can be found in [10].

Along with the dispersive system (42), we consider the hydrostatic model which follows from equations (28)-(33) for $\beta=0$. These equations are also written as a system of balance 
laws in dimensional variables:

$$
\begin{aligned}
& \frac{\partial h}{\partial t}+\frac{\partial}{\partial x}(h U)=-\sigma q, \quad \frac{\partial \eta}{\partial t}+\frac{\partial}{\partial x}(\eta \bar{u})=\sigma q \\
& \frac{\partial}{\partial t}(\bar{\rho} \eta)+\frac{\partial}{\partial x}(\bar{u} \bar{\rho} \eta)=\sigma q \rho_{l}, \quad \frac{\partial}{\partial t}\left(U h+\bar{u} \eta \frac{\bar{\rho}}{\rho_{l}}\right)+ \\
& \frac{\partial}{\partial x}\left(U^{2} h+\left(\bar{u}^{2}+q^{2}\right) \eta \frac{\bar{\rho}}{\rho_{l}}+g h\left(\frac{h}{2}+\frac{\bar{\rho} \eta}{\rho_{l}}\right)+\frac{g \eta^{2} \bar{\rho}}{2 \rho_{l}}\right)=-\left(h+\eta \frac{\bar{\rho}}{\rho_{l}}\right) g b_{x} \\
& \frac{\partial q}{\partial t}+\frac{\partial}{\partial x}(q \bar{u})=\frac{\sigma \rho_{l}}{2 \bar{\rho} \eta}\left((U-\bar{u})^{2}-\left(1+\frac{\kappa \bar{\rho}}{\rho_{l}} \operatorname{sgn}(q)\right) q^{2}-\left(1-\frac{\bar{\rho}}{\rho_{l}}\right) g \eta\right) \\
& \frac{\partial U}{\partial t}+\frac{\partial}{\partial x}\left(\frac{U^{2}}{2}+g\left(h+\frac{\bar{\rho} \eta}{\rho_{l}}\right)\right)=-g b_{x},
\end{aligned}
$$

System (46) belongs to the class of equations (45) with

$$
\mathbf{u}=\left(h, \eta, \bar{\rho} \eta, U h+\bar{u} \eta \bar{\rho} / \rho_{l}, q, U\right)^{\mathrm{T}} .
$$

It was also solved by using the Nessyahu-Tadmor scheme [23].

As in the non-hydrostatic case, we use the equation for 'distortion' variable $q$ (the fifth equation in (46)) instead of the corresponding energy balance law.

The next subsections are devoted to the numerical results.

\subsection{Formation of a stationary turbulent bore over obstacle}

The quasi-steady turbulent bore is modelled on the basis of system (42) and its hydrostatic simplification (46). At $t=0$, the supercritical flow having velocities $U=1.63$ and $\bar{u}=1.75$ in the layers of thickness $h=1$ and $\eta=0.1$ is set. We also suppose that initially $\bar{\rho}=0.9$, and $q=0$. In this example, $g=1, \rho_{l}=1, \sigma=0.15$ and $\kappa=0$. On the left boundary $x=0$, we use the initial data as the boundary conditions; on the right boundary $x=70$, we use the Neumann condition for $\mathbf{u}$. The Nessyahu-Tadmor scheme was applied on a uniform grid of $N=700$ nodes.

The formation of the turbulent bore is due to the variation of the bottom topography. Initially, the bottom is flat $(b=0)$. During time $T_{1}=25$ the amplitude of a smooth obstacle of width 10 located on the right edge is increased to 0.65 , then over time $T_{2}=100$ it is reduced to 0.152 . Such a choice of parameters guarantees the formation of a quasi-steady bore. This is shown in Fig. 5 at $t=500$. We can state that this regime is quasi-steady because there is almost no visible difference in results obtained at $t=300, t=400$, and $t=500$. Curves 1, 2 and 3 correspond to the free surface $z=f$, interface $z=h+b$, and bottom topography $z=b$, respectively. Let us note that the lines 1 and 2 in the region before hydraulic jump $\left(x<x_{c}=38\right)$ are not parallel: we can observe a slight growth of the subsurface shear layer with the increase of $x \in\left(0, x_{c}\right)$. Because of the entrainment of the

pure water into the bubble layer, the fluid velocity (curves 4, 5) substantially decreases. This results in the transition, on average, to the sub-critical flow in the vicinity of the obstacle. The distortion variable $q$ is generated at the wave-front (curve 6 ). This entails intensive 
(a)

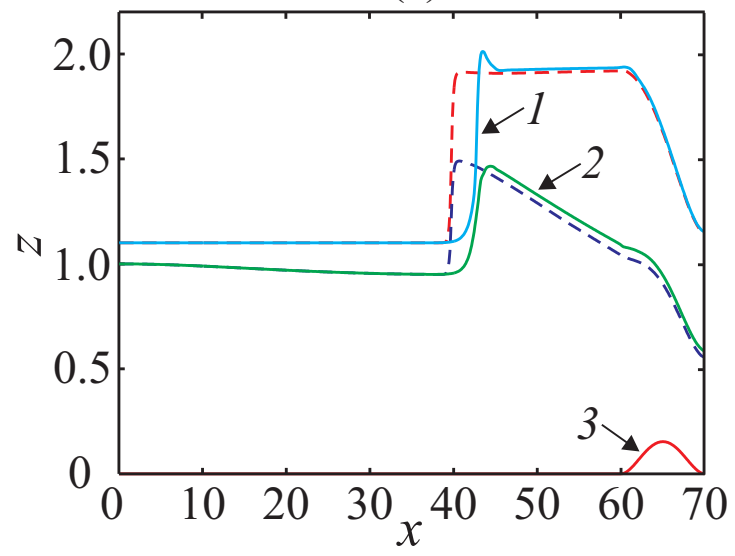

(b)

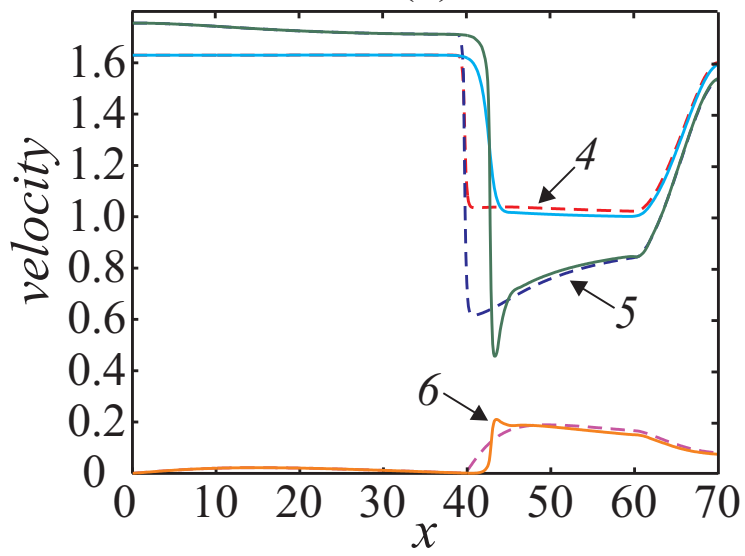

Figure 5: Quasi-steady turbulent bore: (a) shape of the bore (1 - free surface, 2 - internal boundary $z=h+b, 3$ - bottom topography); (b) distribution of the velocities 4 - velocity $U$ in the lower layer, 5 velocity $\bar{u}$ in the upper layer, 6 - variable $q$. Solid curves refer to the solution of Eqs. (42), dashed curves correspond to the solution of the hydrostatic Eqs. (46).

mixing in the region behind the wave-front and leads to the increasing of the fluid density. One can see in Fig. 5 that the position of the wave front is different for the models. Also, in the non-hydrostatic case the wave front behaviour is non-monotonic, while in the hydrostatic case it is monotonic.

\subsection{Formation of periodic and damped oscillations}

As has been shown above, the governing equations for the dissipationless case and flat topography admit periodic stationary solutions. Let us choose the following initial data : $U=0.4, h=1, \bar{\rho}=0.9, \eta=0.1, q=0$, and $\bar{u}=U+\sqrt{\left(1-\rho / \rho_{l}\right) g \eta}=0.5$ at $t=0$, which is an exact solution of equations (42) or (46). At the left boundary of the computational domain $x=0$ we take the initial data as the boundary conditions except for $\bar{u}$, which is equal to 0.54 . This provides inflow of the light liquid in the upper layer through the left boundary. On the right boundary $x=200$, as before, we use the Neumann condition. The computation is carried out for $g=1, \rho_{l}=1$ and $\sigma=0.15$ on a uniform grid of $N=1000$ nodes. We consider two cases corresponding to the presence $(\kappa=1)$ and absence $(\kappa=0)$ of the energy dissipation.

The formation of periodic $(\kappa=0)$ and damped oscillations $(\kappa=1)$ is shown in Fig. 6 at $t=300$ for the dispersive (42) (solid lines) and hydrostatic model (46) (dashed lines). The corresponding stationary solution to (36) is shown by dashed-dotted lines. Numerical results obtained for $\kappa=0(\kappa=1)$ are shown on the left (right) column of the figure. As we can see from Fig. 6, the disturbances generated on the left boundary have not yet reached the right edge of the computational domain at the chosen time moment. This is why near the right boundary we observe significant difference in results obtained on the basis of stationary and non-stationary equations. 


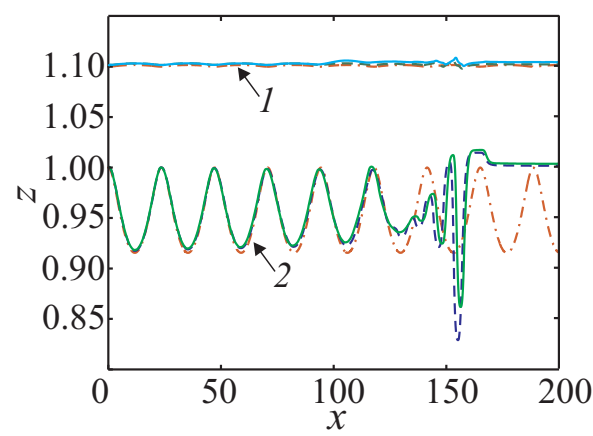

(a)

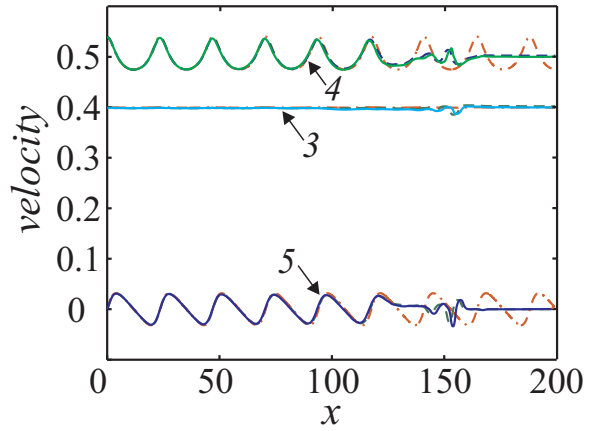

(b)
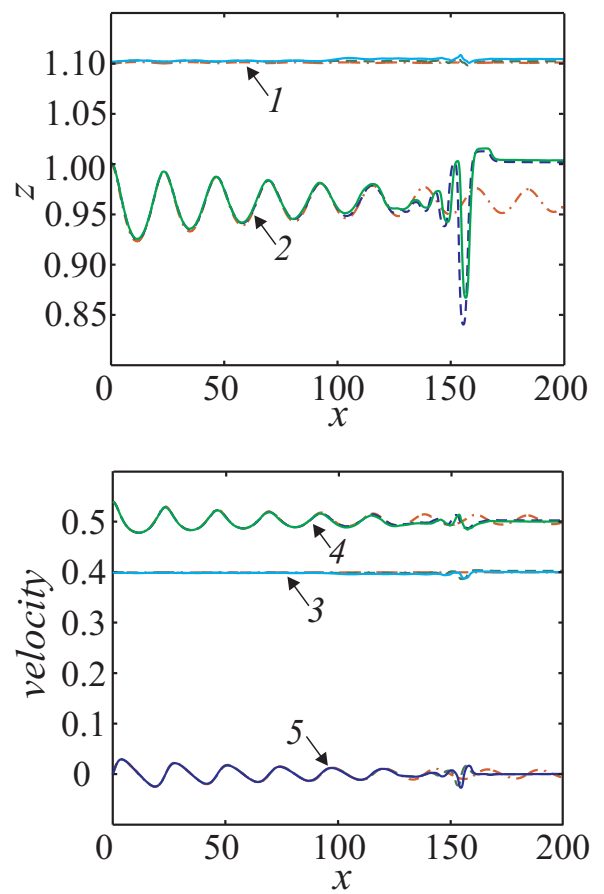

(c)
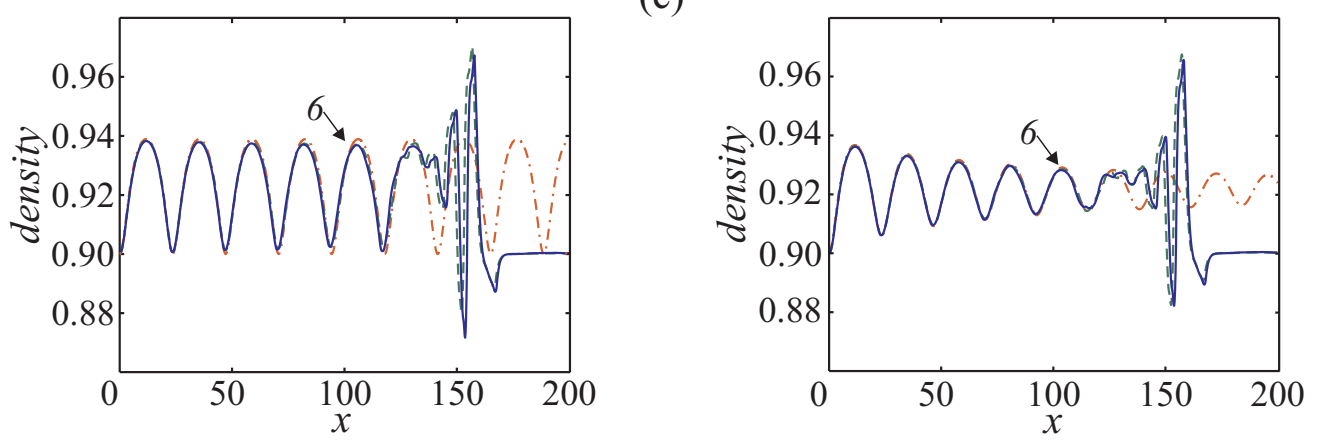

Figure 6: Formation of stationary periodic and damiped in the framework of models (42) (solid lines) and (46) (dashed lines) at $t=300$; dashed-dotted lines correspond to the solution of stationary equations (36): (a) free surface $z=h+\eta$ and interface $z=h$ (curves 1 and 2); (b) distribution of the velocities 3- velocity $U$ in the lower layer, 4 - velocity $\bar{u}$ in the upper layer, 5 - variable q; (c) density distribution (curves 6 ). The graphs are obtained for $\kappa=0$ (on the left) and $\kappa=1$ (on the right). 


\section{Conclusion}

A new two-layer model for the interaction of a bubble shear layer and long internal waves over topography has been derived and presented in a form well suited for numerical simulation. Non-stationary computations were performed to describe the formation of bores, and both periodic and damped oscillations. A hydrostatic approximation of this model was compared to the full non-hydrostatic system. Even if a quantitative description by the both models was quite similar, the qualitative behaviour of wave fronts was different. In particular, a non-monotonic behaviour of bores has been found by using the dispersive model, while it was monotonic in the hydrostatic model.

The analogy between mathematical modeling of two-layer bubble flows and internal waves was underlined. This will allow us to compare in future the theoretical and experimental results. The generation of large amplitude periodic internal waves could be such a case study $[22,13]$.

\section{Appendix A}

The system for the lower layer is:

$$
\begin{gathered}
h_{t}+(h U)_{x}=-M, \\
\frac{\partial}{\partial t}(h U)+\frac{\partial}{\partial x}\left(h U^{2}+P\right)=\left.p\right|_{z=b+h}(b+h)_{x}-\left.p\right|_{z=b} b_{x}-\left.M u\right|_{z=b+h}, \\
\left(h\left(\frac{U^{2}}{2}+e+\frac{h}{2}+b\right)\right)_{t}+\left(h U\left(\frac{U^{2}}{2}+e+\frac{h}{2}+b\right)+U P\right)_{x} \\
=-\left.p\right|_{z=b+h}\left(M+(b+h)_{t}\right)+\left.p\right|_{z=b} b_{t}-\left.M\left(\frac{u^{2}}{2}+\varepsilon^{2} \frac{w^{2}}{2}+h\right)\right|_{z=b+h},
\end{gathered}
$$

where

$$
\begin{gathered}
e=\varepsilon^{2}\left(\frac{1}{2}\left(\frac{D b}{D t}\right)^{2}-\frac{1}{2} \frac{D b}{D t} U_{x} h+\frac{U_{x}^{2} h^{2}}{6}\right), \\
P=\left.p\right|_{z=b+h} h+\frac{h^{2}}{2}+\varepsilon^{2}\left(\frac{h^{2}}{2} \frac{D^{2} b}{D t^{2}}-\frac{h^{3}}{3}\left(\frac{D U_{x}}{D t}-U_{x}^{2}\right)\right), \\
\left.p\right|_{z=b}=\left.p\right|_{z=b+h}+h+\varepsilon^{2} h \frac{D^{2} b}{D t^{2}}-\frac{\varepsilon^{2}}{2} h^{2}\left(\frac{D U_{x}}{D t}-U_{x}^{2}\right) \\
\left.w(t, x, z)\right|_{z=b+h} \approx \frac{D b}{D t}-U_{x} h, \quad \frac{D b}{D t}=b_{t}+U b_{x} .
\end{gathered}
$$

The momentum equation is equivalent to

$$
h \frac{D U}{D t}+P_{x}=\left.p\right|_{z=b+h}(b+h)_{x}-\left.p\right|_{z=b} b_{x}-M\left(\left.u\right|_{z=b+h}-U\right) .
$$


Developing the energy equation by using the momentum equation we have

$$
\begin{gathered}
h \frac{D}{D t}\left(e+\frac{h}{2}+b\right)-M\left(\frac{U^{2}}{2}+e+\frac{h}{2}+b\right)+U_{x} P+ \\
U\left(\left.p\right|_{z=b+h}(b+h)_{x}-\left.p\right|_{z=b} b_{x}-M\left(\left.u\right|_{z=b+h}-U\right)\right)= \\
\quad=-\left.p\right|_{z=b+h}\left(M+(b+h)_{t}\right)+\left.p\right|_{z=b} b_{t}- \\
M\left(\frac{\left.u^{2}\right|_{z=b+h}}{2}+\frac{\varepsilon^{2}}{2}\left(\frac{D b}{D t}-U_{x} h\right)^{2}+b+h\right) .
\end{gathered}
$$

Or

$$
\begin{gathered}
\varepsilon^{2} h \frac{D}{D t}\left(-\frac{1}{2} \frac{D b}{D t} U_{x} h+\frac{U_{x}^{2} h}{6}\right)+U_{x}\left(\varepsilon^{2}\left(\frac{h^{2}}{2} \frac{D^{2} b}{D t^{2}}-\frac{h^{3}}{3}\left(\frac{D U_{x}}{D t}-U_{x}^{2}\right)\right)\right)= \\
\left(-\frac{\varepsilon^{2}}{2} h^{2}\left(\frac{D U_{x}}{D t}-U_{x}^{2}\right)\right) \frac{D b}{D t}- \\
M\left(\frac{\left(\left.u\right|_{z=b+h}-U\right)^{2}}{2}+\varepsilon^{2}\left(-\frac{1}{2} \frac{D b}{D t} U_{x} h+\frac{U_{x}^{2} h}{6}\right)\right) .
\end{gathered}
$$

It is equivalent to

$$
M\left(\left.u\right|_{z=b+h}-U\right)^{2}=0 .
$$

Hence, to be compatible with the energy equation, we need

$$
\left.u\right|_{z=b+h}=U \text {. }
$$

\section{Appendix B}

We consider here the case of time-independent topography $b=b(x)$. In this case $\dot{b}=$ $\frac{D b}{D t}=U b_{x}, \ddot{b}=\frac{D^{2} b}{D t^{2}}=U\left(U b_{x}\right)_{x}$. Consider dimensional form of the momentum equation for

the lower layer. Since $p / \rho_{l}=g \bar{\rho} \eta$, it can be transformed to the equivalent form :

$$
\begin{aligned}
h \frac{D U}{D t}+g h\left(b_{x}\right. & \left.+h_{x}+\left(\frac{\bar{\rho} \eta}{\rho_{l}}\right)_{x}\right)+\left(\frac{h^{2}}{2} \ddot{b}-\frac{h^{3}}{3}\left(U_{x t}+U U_{x x}-U_{x}^{2}\right)\right)_{x}+ \\
& +h\left(\ddot{b}-\frac{h}{2}\left(U_{x t}+U U_{x x}-U_{x}^{2}\right)\right) b_{x}=0 .
\end{aligned}
$$

It is equivalent to :

$$
\begin{gathered}
U_{t}+\left(\frac{U^{2}}{2}+g b+g h+g \frac{\bar{\rho} \eta}{\rho_{l}}\right)_{x} \\
-\left(h h_{x} U_{x t}+\frac{h^{2}}{3} U_{x x t}+h h_{x}\left(U U_{x x}-U_{x}^{2}\right)+\frac{h^{2}}{3}\left(U U_{x x x}-U_{x} U_{x x}\right)\right)
\end{gathered}
$$




$$
+\ddot{b} h_{x}+\frac{h}{2}(\ddot{b})_{x}+\left(\ddot{b}-\frac{h}{2}\left(U_{x t}+U U_{x x}-U_{x}^{2}\right)\right) b_{x}=0 .
$$

It implies :

$$
\begin{gathered}
\left(U-\frac{1}{3 h}\left(h^{3} U_{x}\right)_{x}-\frac{1}{2} h b_{x} U_{x}\right)_{t}+\left(\frac{U^{2}}{2}+g b+g h+g \frac{\bar{\rho} \eta}{\rho_{l}}\right)_{x} \\
-\left(h h_{x}\left(U U_{x x}-U_{x}^{2}\right)+\frac{h^{2}}{3}\left(U U_{x x x}-U_{x} U_{x x}\right)-U_{x} h_{t} h_{x}-U_{x} h h_{t x}-\frac{2}{3} h h_{t} U_{x x}\right) \\
+\ddot{b} h_{x}+\frac{h}{2}(\ddot{b})_{x}+\left(\ddot{b}-\frac{h}{2}\left(U U_{x x}-U_{x}^{2}\right)\right) b_{x}+\frac{1}{2} U_{x} b_{x} h_{t}=0 .
\end{gathered}
$$

Replacing $h_{t}$ by

$$
h_{t}=-(h U)_{x}-M
$$

we obtain :

$$
\begin{gathered}
\left(U-\frac{1}{3 h}\left(h^{3} U_{x}\right)_{x}-\frac{1}{2} h b_{x} U_{x}\right)_{t}+\left(\frac{U^{2}}{2}+g b+g h+g \frac{\bar{\rho} \eta}{\rho_{l}}\right)_{x} \\
-\left(U_{x} h_{x}\left(M+(h U)_{x}\right)+U_{x} h\left(M+(h U)_{x}\right)_{x}+\frac{2}{3} h\left(M+(h U)_{x}\right) U_{x x}\right. \\
\left.+h h_{x}\left(U U_{x x}-U_{x}^{2}\right)+\frac{h^{2}}{3}\left(U U_{x x x}-U_{x} U_{x x}\right)\right) \\
+\ddot{b} h_{x}+\frac{h}{2}(\ddot{b})_{x}+\left(\ddot{b}-\frac{h}{2}\left(U U_{x x}-U_{x}^{2}\right)\right) b_{x}-\frac{1}{2} U_{x} b_{x}\left(M+(h U)_{x}\right)=0 .
\end{gathered}
$$

It can be simplified to :

$$
\begin{gathered}
\left(U-\frac{1}{3 h}\left(h^{3} U_{x}\right)_{x}-\frac{1}{2} h b_{x} U_{x}\right)_{t}+ \\
\left(\frac{U^{2}}{2}+g b+g h+g \frac{\bar{\rho} \eta}{\rho_{l}}-\frac{1}{3 h} U\left(h^{3} U_{x}\right)_{x}-\frac{1}{2} h^{2} U_{x}^{2}\right)_{x}- \\
\left(U_{x}(h M)_{x}+\frac{2}{3} h M U_{x x}\right) \\
+\ddot{b} h_{x}+\frac{h}{2}(\ddot{b})_{x}+\left(\ddot{b}-\frac{h}{2}\left(U U_{x x}-U_{x}^{2}\right)\right) b_{x}-\frac{1}{2} U_{x} b_{x}\left(M+(h U)_{x}\right)=0 .
\end{gathered}
$$

For a slow varying bottom topography one has the equation :

$$
\begin{aligned}
\left(U-\frac{1}{3 h}\left(h^{3} U_{x}\right)_{x}\right)_{t} & +\left(\frac{U^{2}}{2}+g b+g h+g \frac{\bar{\rho} \eta}{\rho_{l}}-\frac{1}{3 h} U\left(h^{3} U_{x}\right)_{x}-\frac{1}{2} h^{2} U_{x}^{2}\right)_{x} \\
& -\left(U_{x}(h M)_{x}+\frac{2}{3} h M U_{x x}\right)=0 .
\end{aligned}
$$

Or, introducing

$$
K=U-\frac{1}{3 h}\left(h^{3} U_{x}\right)_{x}
$$


one obtains

$$
\begin{gathered}
K_{t}+\left(K U-\frac{1}{2}\left(U^{2}+h^{2} U_{x}^{2}\right)+g b+g h+g \frac{\bar{\rho} \eta}{\rho_{l}}\right)_{x}- \\
\left(U_{x}(h M)_{x}+\frac{2}{3} h M U_{x x}\right)=0 .
\end{gathered}
$$

The final form is :

$$
K_{t}+\left(K U-\frac{1}{2}\left(U^{2}+h^{2} U_{x}^{2}\right)+g b+g h+g \frac{\bar{\rho} \eta}{\rho_{l}}-U_{x} h M\right)_{x}=-\frac{1}{3} h M U_{x x} .
$$

The difference with an analogous equation in [10] is the buoyancy term in the flux resulting from the variable density in the subsurface layer.

\section{Acknowledgements}

This work was supported by the Russian Science Foundation (grant No. 15-11-20013). SG has been partially supported by the ANR project BoND (ANR-13-BS01-0009-01).

\section{References}

[1] R. Barros, S. Gavrilyuk, V. Teshukov, Dispersive nonlinear waves in two-layer flows with free surface. I. Model derivation and general properties, Stud. Appl. Math. 119 (2007) 191-211.

[2] M. Brocchini, D.H. Peregrine, The dynamics of strong turbulence at free surfaces. Part 1. Description, J. Fluid Mech. 449 (2001) 225-254.

[3] M. Brocchini, D.H. Peregrine, The dynamics of strong turbulence at free surfaces. Part 2. Free-surface boundary conditions, J. Fluid Mech. 449 (2001) 255-290.

[4] M. Brocchini, Free surface boundary conditions at a bubbly/weakly splashing air-water interface, Phys. Fluids. 14 (2002) 1834-1840.

[5] A. Castro, D. Lannes, Fully nonlinear long-wave models in the presence of vorticity, J. Fluid Mech. 759 (2014) 642-675.

[6] L. Deike, W. K. Melville, S. Popinet, Air entrainment and bubble statistics in breaking waves, J. Fluid. Mech. 801 (2016) 91-129.

[7] J. H. Duncan, Spilling Breakers, Ann. Rev. Fluid Mech. 33 (2001) 519-547.

[8] H. Favre, Ondes de translation dans les canaux découverts, Dunod, Paris, 1935.

[9] S. Gavrilyuk, H. Kalisch, Z. Khorsand, A kinematic conservation law in free surface flow, Nonlinearity 28 (2015) 1805-1821. 
[10] S. L. Gavrilyuk, V. Yu. Liapidevskii, A. A. Chesnokov, Spilling breakers in shallow water: applications to Favre waves and to the shoaling and breaking of solitary waves, J. Fluid Mech. 808 (2016) 441-468.

[11] R. H. J. Grimshaw, K. R. Khusnutdinova, L. A. Ostrovsky, A.S. Topolnikov, Structure formation in the oceanic subsurface bubble layer by an internal wave field, Phys. Fluids. 22 (2010) 106603.

[12] A. E. Green, P. M. Naghdi, A derivation of equations for wave propagation in water of variable depth, J. Fluid Mech. 78 (1976) 237-246.

[13] J. C. Harris, L. Decker, Intermittent large amplitude internal waves observed in Port Susan, Puget Sound, Estuarine Coastal and Shelf Science (2017), doi:10.1016/j.ecss.2017.04.022

[14] S.-H. Hsiao, T.-W. Hsu, T.-C. Lin, Y.-H. Chang, On the evolution and run-up of breaking solitary waves on a mild sloping beach, Coast. Eng. 55 (2008) 975-988.

[15] O. Le Metayer, S. Gavrilyuk, S. Hank, A numerical scheme for the Green-Naghdi model, J. Comput. Phys. 229 (2010) 2034-2045.

[16] V. Yu. Lyapidevskii, V. M. Teshukov, Mathematical Models of Propagation of Long Waves in a Non-Homogeneous Fluid, Siberian Branch of Russian Academy of Sciences, Novosibirsk, 2000. [in Russian]

[17] V. Yu. Liapidevskii, A. A. Chesnokov, Mixing layer under a free surface, J. Appl. Mech. Tech. Phys. 55 (2014) 299-310.

[18] P. Lubin, S. Glockner, Numerical simulations of three-dimensional plunging breaking waves : generation and evolution of aerated vortex filaments, J. Fluid. Mech. 767 (2015) 364-393.

[19] P. A. Madsen, I. A. Svendsen, Turbulent bores and hydraulic jumps, J. Fluid Mech. 129 (1983) $1-25$.

[20] S. K. Misra, M. Brocchini, J. T. Kirby, Turbulent interfacial boundary conditions for spilling breakers, Proc. 30th I.C.C.E. 1 (2006) 214-226.

[21] S. K. Misra, J. T. Kirby, M. Brocchini, F. Veron, M. Thomas, C. Kambhamettu, The mean and turbulent flow structure of a weak hydraulic jump, Phys. Fluids. 20 (2008) 035106 .

[22] J. D. Nash, J. N. Moum, River plumes as a source of large-amplitude internal waves in the coastal ocean, Nature 437 (2005) 400-403.

[23] H. Nessyahu, E. Tadmor, Non-oscillatory central differencing schemes for hyperbolic conservation laws, J. Comp. Phys. 87 (1990) 408-463. 
[24] G. L. Richard, S. L. Gavrilyuk, A new model of roll waves: comparison with Brock's experiments, J. Fluid Mech. 698 (2012) 374-405.

[25] G. L. Richard, S. L. Gavrilyuk, The classical hydraulic jump in a model of shear shallow water flows, J. Fluid Mech. 725 (2013) 492-521.

[26] G. Russo, Central schemes for conservation laws with application to shallow water equations, in S. Rionero, G. Romano (Eds.) Trends and Applications of Mathematics to Mechanics, Springer-Verlag Italia, 2005, pp. 225-246.

[27] F. Serre, Contribution à l'étude des écoulements permanents et variables dans les cannaux, Houille Blanche. 8 (1953 ), 374-388.

[28] C. H. Su, C. S. Gardner, Korteweg - de Vries Equation and Generalisations. III. Derivation of the Korteweg - de Vries Equation and Burgers Equation, J. Math. Physics. 10 (1969) 536-539.

[29] I. A. Svendsen, P. A. Madsen, A turbulent bore on a beach, J. Fluid Mechanics. 148 (1984) 73-96.

[30] V. M. Teshukov, Gas-dynamic analogy for vortex free-boundary flows, J. Appl. Mech. Tech. Phys. 48 (2007) 303-309.

[31] V. M. Teshukov, Gas-dynamic analogy in the theory of stratified liquid flows with a free boundary, Fluid Dynamics 42 (2007) 807-817.

[32] S. A. Thorpe, Bubble clouds and the dynamics of the upper ocean, Quarterly J. Royal Meteorological Society. 118 (1992) 1-22.

[33] A. A. Townsend, 1956 The structure of turbulent shear flow, Cambridge University Press. 